\title{
The $e^{+} e^{-} \rightarrow 3\left(\pi^{+} \pi^{-}\right), 2\left(\pi^{+} \pi^{-} \pi^{0}\right)$ and $K^{+} K^{-} 2\left(\pi^{+} \pi^{-}\right)$cross sections at center-of-mass energies from production threshold to $4.5 \mathrm{GeV}$ measured with initial-state radiation
}

B. Aubert, R. Barate, D. Boutigny, F. Couderc, Y. Karyotakis, J. P. Lees, V. Poireau, V. Tisserand, and A. Zghiche Laboratoire de Physique des Particules, F-74941 Annecy-le-Vieux, France

E. Grauges

IFAE, Universitat Autonoma de Barcelona, E-08193 Bellaterra, Barcelona, Spain

A. Palano and M. Pappagallo

Università di Bari, Dipartimento di Fisica and INFN, I-70126 Bari, Italy

J. C. Chen, N. D. Qi, G. Rong, P. Wang, and Y. S. Zhu

Institute of High Energy Physics, Beijing 100039, China

G. Eigen, I. Ofte, and B. Stugu

University of Bergen, Institute of Physics, N-5007 Bergen, Norway

G. S. Abrams, M. Battaglia, D. S. Best, D. N. Brown, J. Button-Shafer, R. N. Cahn, E. Charles, C. T. Day, M. S. Gill, A. V. Gritsan, * Y. Groysman, R. G. Jacobsen, R. W. Kadel, J. A. Kadyk, L. T. Kerth, Yu. G. Kolomensky, G. Kukartsev, G. Lynch, L. M. Mir, P. J. Oddone, T. J. Orimoto, M. Pripstein, N. A. Roe, M. T. Ronan, and W. A. Wenzel Lawrence Berkeley National Laboratory and University of California, Berkeley, California 94720, USA

M. Barrett, K. E. Ford, T. J. Harrison, A. J. Hart, C. M. Hawkes, S. E. Morgan, and A. T. Watson University of Birmingham, Birmingham, B15 2TT, United Kingdom

M. Fritsch, K. Goetzen, T. Held, H. Koch, B. Lewandowski, M. Pelizaeus, K. Peters, T. Schroeder, and M. Steinke Ruhr Universität Bochum, Institut für Experimentalphysik 1, D-44780 Bochum, Germany

J. T. Boyd, J. P. Burke, W. N. Cottingham, and D. Walker University of Bristol, Bristol BS8 1TL, United Kingdom

T. Cuhadar-Donszelmann, B. G. Fulsom, C. Hearty, N. S. Knecht, T. S. Mattison, and J. A. McKenna University of British Columbia, Vancouver, British Columbia, Canada V6T 1Z1

A. Khan, P. Kyberd, M. Saleem, and L. Teodorescu Brunel University, Uxbridge, Middlesex UB8 3PH, United Kingdom

V.E. Blinov, A. D. Bukin, V. P. Druzhinin, V. B. Golubev, E. A. Kravchenko, A. P. Onuchin, S. I. Serednyakov, Yu. I. Skovpen, E. P. Solodov, and K. Yu Todyshev Budker Institute of Nuclear Physics, Novosibirsk 630090, Russia

M. Bondioli, M. Bruinsma, M. Chao, S. Curry, I. Eschrich, D. Kirkby, A. J. Lankford, P. Lund, M. Mandelkern,

R. K. Mommsen, W. Roethel, and D. P. Stoker University of California at Irvine, Irvine, California 92697, USA

S. Abachi and C. Buchanan University of California at Los Angeles, Los Angeles, California 90024, USA

S. D. Foulkes, J. W. Gary, O. Long, B. C. Shen, K. Wang, and L. Zhang University of California at Riverside, Riverside, California 92521, USA

D. del Re, H. K. Hadavand, E. J. Hill, H. P. Paar, S. Rahatlou, and V. Sharma University of California at San Diego, La Jolla, California 92093, USA

J. W. Berryhill, C. Campagnari, A. Cunha, B. Dahmes, T. M. Hong, and J. D. Richman University of California at Santa Barbara, Santa Barbara, California 93106, USA 
T. W. Beck, A. M. Eisner, C. J. Flacco, C. A. Heusch, J. Kroseberg, W. S. Lockman, G. Nesom, T. Schalk, B. A. Schumm, A. Seiden, P. Spradlin, D. C. Williams, and M. G. Wilson University of California at Santa Cruz, Institute for Particle Physics, Santa Cruz, California 95064, USA

J. Albert, E. Chen, G. P. Dubois-Felsmann, A. Dvoretskii, D. G. Hitlin, I. Narsky, T. Piatenko, F. C. Porter, A. Ryd, and A. Samuel California Institute of Technology, Pasadena, California 91125, USA

R. Andreassen, G. Mancinelli, B. T. Meadows, and M. D. Sokoloff University of Cincinnati, Cincinnati, Ohio 45221, USA

F. Blanc, P. C. Bloom, S. Chen, W. T. Ford, J. F. Hirschauer, A. Kreisel, U. Nauenberg, A. Olivas, W. O. Ruddick, J. G. Smith, K. A. Ulmer, S. R. Wagner, and J. Zhang University of Colorado, Boulder, Colorado 80309, USA

A. Chen, E. A. Eckhart, A. Soffer, W. H. Toki, R. J. Wilson, F. Winklmeier, and Q. Zeng Colorado State University, Fort Collins, Colorado 80523, USA

D. D. Altenburg, E. Feltresi, A. Hauke, H. Jasper, and B. Spaan Universität Dortmund, Institut für Physik, D-44221 Dortmund, Germany

T. Brandt, M. Dickopp, V. Klose, H. M. Lacker, R. Nogowski, S. Otto, A. Petzold, J. Schubert, K. R. Schubert, R. Schwierz, J.E. Sundermann, and A. Volk Technische Universität Dresden, Institut für Kern- und Teilchenphysik, D-01062 Dresden, Germany

D. Bernard, G. R. Bonneaud, P. Grenier, ${ }^{\dagger}$ E. Latour, S. Schrenk, Ch. Thiebaux, G. Vasileiadis, and M. Verderi Ecole Polytechnique, LLR, F-91128 Palaiseau, France

D. J. Bard, P. J. Clark, W. Gradl, F. Muheim, S. Playfer, and Y. Xie University of Edinburgh, Edinburgh EH9 3JZ, United Kingdom

M. Andreotti, D. Bettoni, C. Bozzi, R. Calabrese, G. Cibinetto, E. Luppi, M. Negrini, and L. Piemontese Università di Ferrara, Dipartimento di Fisica and INFN, I-44100 Ferrara, Italy

F. Anulli, R. Baldini-Ferroli, A. Calcaterra, R. de Sangro, G. Finocchiaro, S. Pacetti, P. Patteri, I. M. Peruzzi, ${ }^{\ddagger}$ M. Piccolo, and A. Zallo Laboratori Nazionali di Frascati dell'INFN, I-00044 Frascati, Italy

A. Buzzo, R. Capra, R. Contri, M. Lo Vetere, M. M. Macri, M. R. Monge, S. Passaggio, C. Patrignani, E. Robutti, A. Santroni, and S. Tosi Università di Genova, Dipartimento di Fisica and INFN, I-16146 Genova, Italy

G. Brandenburg, K. S. Chaisanguanthum, M. Morii, and J. Wu Harvard University, Cambridge, Massachusetts 02138, USA

R. S. Dubitzky, J. Marks, S. Schenk, and U. Uwer Universität Heidelberg, Physikalisches Institut, Philosophenweg 12, D-69120 Heidelberg, Germany

W. Bhimji, D. A. Bowerman, P. D. Dauncey, U. Egede, R. L. Flack, J. R. Gaillard, J . A. Nash, M. B. Nikolich, and W. Panduro Vazquez Imperial College London, London, SW7 2AZ, United Kingdom

X. Chai, M. J. Charles, W. F. Mader, U. Mallik, and V. Ziegler University of Iowa, Iowa City, Iowa 52242, USA

J. Cochran, H. B. Crawley, L. Dong, V. Eyges, W. T. Meyer, S. Prell, E. I. Rosenberg, and A. E. Rubin Iowa State University, Ames, Iowa 50011-3160, USA 


\section{G. Schott}

Universität Karlsruhe, Institut für Experimentelle Kernphysik, D-76021 Karlsruhe, Germany

N. Arnaud, M. Davier, G. Grosdidier, A. Höcker, F. Le Diberder, V. Lepeltier, A. M. Lutz, A. Oyanguren, T. C. Petersen, S. Pruvot, S. Rodier, P. Roudeau, M. H. Schune, A. Stocchi, W. F. Wang, and G. Wormser

Laboratoire de l'Accélérateur Linéaire, F-91898 Orsay, France

C. H. Cheng, D. J. Lange, and D. M. Wright

Lawrence Livermore National Laboratory, Livermore, California 94550, USA

A. J. Bevan, C. A. Chavez, I. J. Forster, J. R. Fry, E. Gabathuler, R. Gamet, K. A. George, D. E. Hutchcroft, D. J. Payne, K. C. Schofield, and C. Touramanis

University of Liverpool, Liverpool L69 7ZE, United Kingdom

F. Di Lodovico, W. Menges, and R. Sacco

Queen Mary, University of London, E1 4NS, United Kingdom

C. L. Brown, G. Cowan, H. U. Flaecher, M. G. Green, D. A. Hopkins, P. S. Jackson, T. R. McMahon,

S. Ricciardi, and F. Salvatore

University of London, Royal Holloway and Bedford New College, Egham, Surrey TW20 OEX, United Kingdom

D. N. Brown and C. L. Davis

University of Louisville, Louisville, Kentucky 40292, USA

J. Allison, N. R. Barlow, R. J. Barlow, Y. M. Chia, C. L. Edgar, M. P. Kelly, G. D. Lafferty, M. T. Naisbit, J.C. Williams, and J. I. Yi

University of Manchester, Manchester M13 9PL, United Kingdom

C. Chen, W. D. Hulsbergen, A. Jawahery, D. Kovalskyi, C. K. Lae, D. A. Roberts, and G. Simi University of Maryland, College Park, Maryland 20742, USA

G. Blaylock, C. Dallapiccola, S. S. Hertzbach, R. Kofler, X. Li, T. B. Moore, S. Saremi, H. Staengle, and S. Y. Willocq University of Massachusetts, Amherst, Massachusetts 01003, USA

R. Cowan, K. Koeneke, G. Sciolla, S. J. Sekula, M. Spitznagel, F. Taylor, and R. K. Yamamoto Massachusetts Institute of Technology, Laboratory for Nuclear Science, Cambridge, Massachusetts 02139, USA

H. Kim, P. M. Patel, C. T. Potter, and S. H. Robertson

McGill University, Montréal, Québec, Canada H3A 2T8

A. Lazzaro, V. Lombardo, and F. Palombo

Università di Milano, Dipartimento di Fisica and INFN, I-20133 Milano, Italy

J. M. Bauer, L. Cremaldi, V. Eschenburg, R. Godang, R. Kroeger, J. Reidy, D. A. Sanders, D. J. Summers, and H. W. Zhao University of Mississippi, University, Mississippi 38677, USA

S. Brunet, D. Côté, P. Taras, and F. B. Viaud

Université de Montréal, Physique des Particules, Montréal, Québec, Canada H3C 3J7

H. Nicholson

Mount Holyoke College, South Hadley, Massachusetts 01075, USA

N. Cavallo, ${ }^{\S}$ G. De Nardo, F. Fabozzi,${ }^{\S}$ C. Gatto, L. Lista, D. Monorchio, P. Paolucci, D. Piccolo, and C. Sciacca Università di Napoli Federico II, Dipartimento di Scienze Fisiche and INFN, I-80126, Napoli, Italy

M. Baak, H. Bulten, G. Raven, and H. L. Snoek

NIKHEF, National Institute for Nuclear Physics and High Energy Physics, NL-1009 DB Amsterdam, The Netherlands 


\author{
C. P. Jessop and J. M. LoSecco \\ University of Notre Dame, Notre Dame, Indiana 46556, USA
}

T. Allmendinger, G. Benelli, K. K. Gan, K. Honscheid, D. Hufnagel, P. D. Jackson, H. Kagan, R. Kass, T. Pulliam, A. M. Rahimi, R. Ter-Antonyan, and Q. K. Wong Ohio State University, Columbus, Ohio 43210, USA

N. L. Blount, J. Brau, R. Frey, O. Igonkina, M. Lu, R. Rahmat, N. B. Sinev, D. Strom, J. Strube, and E. Torrence University of Oregon, Eugene, Oregon 97403, USA

F. Galeazzi, M. Margoni, M. Morandin, A. Pompili, M. Posocco, M. Rotondo, F. Simonetto, R. Stroili, and C. Voci Università di Padova, Dipartimento di Fisica and INFN, I-35131 Padova, Italy

M. Benayoun, J. Chauveau, P. David, L. Del Buono, Ch. de la Vaissière, O. Hamon, B. L. Hartfiel, M. J. J. John, Ph. Leruste, J. Malclès, J. Ocariz, L. Roos, and G. Therin Universités Paris VI et VII, Laboratoire de Physique Nucléaire et de Hautes Energies, F-75252 Paris, France

P. K. Behera, L. Gladney, and J. Panetta University of Pennsylvania, Philadelphia, Pennsylvania 19104, USA

M. Biasini, R. Covarelli, and M. Pioppi Università di Perugia, Dipartimento di Fisica and INFN, I-06100 Perugia, Italy

C. Angelini, G. Batignani, S. Bettarini, F. Bucci, G. Calderini, M. Carpinelli, R. Cenci, F. Forti, M. A. Giorgi, A. Lusiani, G. Marchiori, M. A. Mazur, M. Morganti, N. Neri, E. Paoloni, M. Rama, G. Rizzo, and J. Walsh Università di Pisa, Dipartimento di Fisica, Scuola Normale Superiore and INFN, I-56127 Pisa, Italy

M. Haire, D. Judd, and D. E. Wagoner Prairie View A\&M University, Prairie View, Texas 77446, USA

J. Biesiada, N. Danielson, P. Elmer, Y. P. Lau, C. Lu, J. Olsen, A. J. S. Smith, and A. V. Telnov Princeton University, Princeton, New Jersey 08544, USA

F. Bellini, G. Cavoto, A. D’Orazio, E. Di Marco, R. Faccini, F. Ferrarotto, F. Ferroni, M. Gaspero, L. Li Gioi, M. A. Mazzoni, S. Morganti, G. Piredda, F. Polci, F. Safai Tehrani, and C. Voena Università di Roma La Sapienza, Dipartimento di Fisica and INFN, I-00185 Roma, Italy

\author{
H. Schröder and R. Waldi \\ Universität Rostock, D-18051 Rostock, Germany
}

T. Adye, N. De Groot, B. Franek, E. O. Olaiya, and F. F. Wilson Rutherford Appleton Laboratory, Chilton, Didcot, Oxon, OX11 OQX, United Kingdom

S. Emery, A. Gaidot, S. F. Ganzhur, G. Hamel de Monchenault, W. Kozanecki, M. Legendre, B. Mayer, G. Vasseur, Ch. Yèche, and M. Zito DSM/Dapnia, CEA/Saclay, F-91191 Gif-sur-Yvette, France

W. Park, M. V. Purohit, A. W. Weidemann, and J. R. Wilson University of South Carolina, Columbia, South Carolina 29208, USA

M. T. Allen, D. Aston, R. Bartoldus, N. Berger, A. M. Boyarski, R. Claus, J. P. Coleman, M. R. Convery, M. Cristinziani, J. C. Dingfelder, D. Dong, J. Dorfan, D. Dujmic, W. Dunwoodie, R. C. Field, T. Glanzman, S. J. Gowdy, V. Halyo, C. Hast, T. Hryn'ova, W. R. Innes, M. H. Kelsey, P. Kim, M. L. Kocian, D. W. G. S. Leith, J. Libby, S. Luitz, V. Luth, H. L. Lynch, D. B. MacFarlane, H. Marsiske, R. Messner, D. R. Muller, C. P. O’Grady, V. E. Ozcan, A. Perazzo, M. Perl, B. N. Ratcliff, A. Roodman, A. A. Salnikov, R. H. Schindler, J. Schwiening, A. Snyder, J. Stelzer, D. Su, M. K. Sullivan, K. Suzuki, 
THE $e^{+} e^{-} \rightarrow 3\left(\pi^{+} \pi^{-}\right), 2\left(\pi^{+} \pi^{-} \pi^{0}\right)$ AND $K^{+} K^{-} 2\left(\pi^{+} \pi^{-}\right) \ldots$

PHYSICAL REVIEW D 73, 052003 (2006)

S. K. Swain, J. M. Thompson, J. Va'vra, N. van Bakel, M. Weaver, A. J. R. Weinstein, W. J. Wisniewski, M. Wittgen, D. H. Wright, A. K. Yarritu, K. Yi, and C. C. Young Stanford Linear Accelerator Center, Stanford, California 94309, USA

\section{P. R. Burchat, A. J. Edwards, S. A. Majewski, B. A. Petersen, C. Roat, and L. Wilden} Stanford University, Stanford, California 94305-4060, USA

S. Ahmed, M. S. Alam, R. Bula, J. A. Ernst, V. Jain, B. Pan, M. A. Saeed, F. R. Wappler, and S. B. Zain State University of New York, Albany, New York 12222, USA

W. Bugg, M. Krishnamurthy, and S. M. Spanier University of Tennessee, Knoxville, Tennessee 37996, USA

R. Eckmann, J. L. Ritchie, A. Satpathy, and R.F. Schwitters University of Texas at Austin, Austin, Texas 78712, USA

J. M. Izen, I. Kitayama, X. C. Lou, and S. Ye University of Texas at Dallas, Richardson, Texas 75083, USA

F. Bianchi, M. Bona, F. Gallo, and D. Gamba Università di Torino, Dipartimento di Fisica Sperimentale and INFN, I-10125 Torino, Italy

M. Bomben, L. Bosisio, C. Cartaro, F. Cossutti, G. Della Ricca, S. Dittongo, S. Grancagnolo, L. Lanceri, and L. Vitale Università di Trieste, Dipartimento di Fisica and INFN, I-34127 Trieste, Italy

V. Azzolini and F. Martinez-Vidal

IFIC, Universitat de Valencia-CSIC, E-46071 Valencia, Spain

R. S. Panvini"l

Vanderbilt University, Nashville, Tennessee 37235, USA

Sw. Banerjee, B. Bhuyan, C. M. Brown, D. Fortin, K. Hamano, R. Kowalewski, I. M. Nugent, J. M. Roney, and R. J. Sobie University of Victoria, Victoria, British Columbia, Canada V8W $3 P 6$

J. J. Back, P. F. Harrison, T. E. Latham, and G. B. Mohanty Department of Physics, University of Warwick, Coventry CV4 7AL, United Kingdom

H. R. Band, X. Chen, B. Cheng, S. Dasu, M. Datta, A. M. Eichenbaum, K. T. Flood, M. T. Graham, J. J. Hollar, J. R. Johnson, P. E. Kutter, H. Li, R. Liu, B. Mellado, A. Mihalyi, A. K. Mohapatra, Y. Pan, M. Pierini, R. Prepost, P. Tan, S. L. Wu, and Z. Yu

University of Wisconsin, Madison, Wisconsin 53706, USA

H. Neal

Yale University, New Haven, Connecticut 06511, USA

(Received 3 February 2006; published 7 March 2006)

We study the processes $e^{+} e^{-} \rightarrow 3\left(\pi^{+} \pi^{-}\right) \gamma, 2\left(\pi^{+} \pi^{-} \pi^{0}\right) \gamma$ and $K^{+} K^{-} 2\left(\pi^{+} \pi^{-}\right) \gamma$, with the photon radiated from the initial state. About 20000,33000 and 4000 fully reconstructed events, respectively, have been selected from $232 \mathrm{fb}^{-1}$ of $B A B A R$ data. The invariant mass of the hadronic final state defines the effective $e^{+} e^{-}$center-of-mass energy, so that these data can be compared with the corresponding direct $e^{+} e^{-}$measurements. From the $3\left(\pi^{+} \pi^{-}\right), 2\left(\pi^{+} \pi^{-} \pi^{0}\right)$ and $K^{+} K^{-} 2\left(\pi^{+} \pi^{-}\right)$mass spectra, the cross sections for the processes $e^{+} e^{-} \rightarrow 3\left(\pi^{+} \pi^{-}\right), e^{+} e^{-} \rightarrow 2\left(\pi^{+} \pi^{-} \pi^{0}\right)$ and $e^{+} e^{-} \rightarrow K^{+} K^{-} 2\left(\pi^{+} \pi^{-}\right)$are measured for center-of-mass energies from production threshold to $4.5 \mathrm{GeV}$. The uncertainty in the cross section measurement is typically $6 \%-15 \%$. We observe a structure at $1.9 \mathrm{GeV}$ in both cross sections and a resonance structure with mass $1645 \pm 0.008 \mathrm{GeV} / \mathrm{c}^{2}$ and width $0.114 \pm 0.014 \mathrm{GeV}$ when the $\omega(782) \eta$ final state is extracted. We observe the $J / \psi$ in all these final states and measure the corresponding branching fractions.

DOI: 10.1103/PhysRevD.73.052003

PACS numbers: 13.66.Bc, 13.25.Gv, 13.25.Jx, 14.40.Cs 


\section{INTRODUCTION}

The idea of utilizing initial-state radiation (ISR) from a high-mass state to explore electron-positron processes at all energies below that state was outlined in Ref. [1]. The possibility of exploiting such processes in high luminosity $\phi$ - and $B$ factories was discussed in Refs. [2-4] and motivates the hadronic cross section measurement described in this paper. This is of particular interest because of the small deviation of the measured muon $g-2$ value from that predicted by the standard model [5], where hadronic loop contributions are obtained from $e^{+} e^{-}$experiments at low center-of-mass (c.m.) energies. The study of ISR events at $B$ factories provides independent and contiguous measurements of hadronic cross sections in this energy region and also contributes to the investigation of low-mass resonance spectroscopy.

The ISR cross section for a particular hadronic final state $f$ is related to the corresponding $e^{+} e^{-}$cross section $\sigma_{f}(s)$ by

$$
\frac{d \sigma_{f}(s, x)}{d x}=W(s, x) \cdot \sigma_{f}(s(1-x))
$$

where $x=2 E_{\gamma} / \sqrt{s} ; E_{\gamma}$ is the energy of the ISR photon in the nominal $e^{+} e^{-}$c.m. frame; $\sqrt{s}=E_{\text {c.m. }}$ is the nominal $e^{+} e^{-}$c.m. energy; and $\sqrt{s(1-x)}$ is the effective c.m. energy at which the final state $f$ is produced. The invariant mass of the hadronic final state is used to measure the effective $e^{+} e^{-}$c.m. energy. The function $W(s, x)$ is calculated with better than $1 \%$ accuracy (see, for example, Ref. [4]) and describes the probability density function for ISR photon emission. ISR photons are produced at all angles, with a distribution peaking at small angles with respect to the axis of the beams, and are required to be detected in the electromagnetic calorimeter (EMC) of the $B A B A R$ detector. The acceptance for such photons is $10 \%-$ $15 \%$ [4] depending on applied selections.

An important advantage of ISR data is that the entire range of effective c.m. energies is scanned in one experiment. This avoids the relative normalization uncertainties that inevitably arise when data from different experiments, or from different machine settings, are combined.

A disadvantage of the ISR measurement is that the mass resolution is much poorer than can be obtained in direct annihilation. The resolution and absolute energy scale can be monitored directly using the measured width and mass

\footnotetext{
*Also with the Johns Hopkins University, Baltimore, MD 21218, USA.

${ }^{\dagger}$ Also at Laboratoire de Physique Corpusculaire, ClermontFerrand, France.

¥Also with Università di Perugia, Dipartimento di Fisica, Perugia, Italy.

${ }^{\S}$ Also with Università della Basilicata, Potenza, Italy.

"Deceased.
}

of the $J / \psi$ resonance produced in the reaction $e^{+} e^{-} \rightarrow$ $J / \psi \gamma$. Using a kinematic fit to this reaction, we find the resolution to be about $9 \mathrm{MeV} / \mathrm{c}^{2}$ for decays of $J / \psi$ in the $3\left(\pi^{+} \pi^{-}\right)$mode and about $15 \mathrm{MeV} / \mathrm{c}^{2}$ in the $2\left(\pi^{+} \pi^{-} \pi^{0}\right)$ mode as will be shown later.

Studies of $e^{+} e^{-} \rightarrow \mu^{+} \mu^{-} \gamma$ and several multihadron ISR processes using BABAR data have been reported previously [6-8]. These demonstrated good detector efficiency and particle identification capability for events of this kind.

This paper reports analyses of the $3\left(\pi^{+} \pi^{-}\right)$, $2\left(\pi^{+} \pi^{-} \pi^{0}\right)$ and $K^{+} K^{-} 2\left(\pi^{+} \pi^{-}\right)$final states produced in conjunction with a hard photon, assumed to result from ISR. A clear $J / \psi$ signal is observed for each of these hadronic final states and the corresponding $J / \psi$ branching fractions are measured. While $B A B A R$ data are available at effective c.m. energies up to $10.58 \mathrm{GeV}$, the present analysis is restricted to energies below $4.5 \mathrm{GeV}$ because of the increase with energy of the backgrounds from non-ISR multihadron production.

\section{THE BABAR DETECTOR AND DATA SET}

The data used in this analysis were collected with the $B A B A R$ detector at the PEP-II asymmetric $e^{+} e^{-}$storage ring. The total integrated luminosity used is $232 \mathrm{fb}^{-1}$, which includes data collected at the $Y(4 S)$ resonance mass $\left(211 \mathrm{fb}^{-1}\right)$, and at a c.m. energy $40 \mathrm{MeV}$ lower $\left(21 \mathrm{fb}^{-1}\right)$.

The $B A B A R$ detector is described elsewhere [9]. Charged particles are reconstructed in the BABAR tracking system, which comprises the silicon vertex tracker (SVT) and the drift chamber (DCH). Separation of pions and kaons is accomplished by means of the detector of internally reflected Cherenkov light (DIRC) and energy-loss measurements in the SVT and DCH. The hard ISR photon and photons from $\pi^{0}$ decays are detected in the electromagnetic calorimeter (EMC). Muon identification is provided by the instrumented flux return (IFR).

The initial selection of candidate events requires that a high-energy photon in the event with $E_{\mathrm{c} . \mathrm{m} .}^{\gamma}>3 \mathrm{GeV}$ be found recoiling against six good-quality charged tracks with zero net charge or against four good-quality charged tracks with zero net charge and four or more photons with energy higher than $0.02 \mathrm{GeV}$. Almost every candidate event has extra soft photons with energy above this threshold, mostly due to secondary hadron interactions and machine background. Each charged track is required to originate close to the interaction region, to have transverse momentum greater than $0.1 \mathrm{GeV} / \mathrm{c}$ and to have a polar angle in the laboratory frame with respect to the collision axis in the range from 0.4 to $2.45 \mathrm{rad}$. These selections guarantee the quality of the charged tracks in the DCH. The chargedtrack vertex is used as the point of origin to calculate the angles for all detected photons. Events with electrons and positrons are removed on the basis of associated EMC 
energy deposition and energy-loss $(\mathrm{d} E / \mathrm{d} x)$ information from the DCH.

In order to study the detector acceptance and efficiency, we developed a set of simulation programs for radiative processes. The simulation of the $3\left(\pi^{+} \pi^{-}\right) \gamma$ and $2\left(\pi^{+} \pi^{-} \pi^{0}\right) \gamma$ final states is based on the generator developed according to the approach suggested by Kühn and Czyż [10]. For the acceptance study we simulate sixcharged pions in a phase space model and in a model which assumes only one $\rho(770)$ per event, i.e. a $\rho(770) 2\left(\pi^{+} \pi^{-}\right)$final state. The $2\left(\pi^{+} \pi^{-} \pi^{0}\right)$ and $K^{+} K^{-} 2\left(\pi^{+} \pi^{-}\right)$final states are simulated according to phase space.

Multiple soft-photon emission from the initial-state charged particles is implemented with the structurefunction technique $[11,12]$, while extra photon radiation from the final-state particles is simulated by means of the PHOTOS package [13]. The accuracy of the radiative corrections is about $1 \%$.

A sample of about 400000 events was generated with these tools for each mode and passed through the detector response simulation [14]. These events were then reconstructed through the same software chain as the experimental data. Variations in detector and background conditions were taken into account.

For purposes of background estimation, a large sample of events from the main ISR processes $(2 \pi \gamma, 3 \pi \gamma, \ldots$, $5 \pi \gamma, 2 K \pi \gamma, \ldots)$ was simulated. This sample exceeded the expected number of events in the data set by a factor of about three. In addition, the expected numbers of $e^{+} e^{-} \rightarrow q \bar{q}(q=u, d, s, c)$ events were generated via JETSET [15] and $e^{+} e^{-} \rightarrow \tau^{+} \tau^{-}$via KORALB [16] in order to estimate background contributions from non-ISR events. The cross sections for the above processes are known with about $10 \%$ accuracy or better, which is sufficient for the background contribution study.

\section{THE KINEMATIC FIT PROCEDURE}

The initial sample of candidate events is subjected to a constrained kinematic fit in conjunction with chargedparticle identification to extract events corresponding to the final states of interest.

For each particular six-charged-particle candidate, and for each possible combination of particle types [i.e. $3\left(\pi^{+} \pi^{-}\right)$or $K^{+} K^{-} 2\left(\pi^{+} \pi^{-}\right)$], a one-constraint (1C) kinematic fit is performed without using information from the detected photon candidate. The only constraint used is zero photon mass. Because of the excellent resolution of the $\mathrm{DCH}$, the three-momentum vector of the photon is better determined through momentum conservation than through measurement in the EMC. As a consequence, the calibration accuracy of the EMC and its alignment with respect to the DCH do not contribute to the systematic uncertainties. The initial $e^{+} e^{-}$and final-state charged-particle four- momenta and their covariance matrices are taken into account.

The fit for the six-pion final-state hypothesis is retained for every event. If only one track is identified as a kaon, or if two oppositely charged kaons are identified, the $K^{+} K^{-} 2\left(\pi^{+} \pi^{-}\right)$fit is also retained.

For the $2\left(\pi^{+} \pi^{-} \pi^{0}\right) \gamma$ events a kinematic fit is performed using the initial $e^{+} e^{-}$, final-state charged-particle and photon four-momenta and their covariance matrices. The highest c.m. energy photon is assumed to be from ISR. Only the direction of the photon momentum vector is used in the fit, not the measured energy. All other photons with energies above $20 \mathrm{MeV}$ are paired. Combinations lying within $\pm 35 \mathrm{MeV} / \mathrm{c}^{2}$ of the $\pi^{0}$ mass are tested, and the event combination with the best $\chi^{2}$ value is retained, subject to the additional constraint that the two, twophoton pairs are consistent with the $\pi^{0}$ mass. In total five constraints (5C fit) are applied. The three-momentum vectors obtained from the fit for each charged track and photon are used in further calculations.

\section{THE $3\left(\pi^{+} \pi^{-}\right)$FINAL STATE}

\section{A. Additional selection criteria}

The results of the $1 \mathrm{C}$ fit to the six-charged-track candidates are used to make the final selection of the six-pion sample. The momentum vector of the photon reconstructed by the fit in the laboratory frame is required to have a polar angle $\theta_{\gamma}^{\text {fit }}$ in the range from 0.35 to $2.4 \mathrm{rad}$ and to match the measured polar angle $\theta_{\gamma}^{\text {meas }}$ of the ISR photon in the EMC within $50 \mathrm{mrad}$. The corresponding azimuthal angles, $\phi_{\gamma}^{\mathrm{fit}}$ and $\phi_{\gamma}^{\text {meas }}$, are also required to agree within this same tolerance. These angular criteria reduce the background by a factor of about two with no noticeable loss of signal. Finally, the polar angle $\theta_{\mathrm{ch}}^{\mathrm{fit}}$ of each charged track obtained from the fit has to satisfy $0.45<\theta_{\mathrm{ch}}^{\mathrm{fit}}<2.4 \mathrm{rad}$ in order to fall within the acceptance of the DIRC, which provides about $80 \%$ of the kaon identification efficiency.

The 1C-fit $\chi^{2}$ distribution for the six-pion candidates is shown as the upper histogram of Fig. 1, while the shaded region is for the corresponding MC-simulated pure $6 \pi \gamma$ events. The experimental distribution has a contribution from background processes, but the pure $6 \pi \gamma \mathrm{MC}$ simulated distribution is also much broader than the usual one-constraint $\chi^{2}$ distribution. This is due to multiple softphoton emission (detected or not detected) in the initial state and radiation from the final-state charged particles, neither of which is taken into account by the constrained fit but which exist both in the data and the MC simulation. The MC-simulated $\chi^{2}$ distribution of Fig. 1 is normalized to the data in the region $\chi^{2}<1$ where the background contamination and multiple soft-photon emission due to ISR or FSR is lowest.

The cross-hatched histogram in Fig. 1 represents the non-ISR background contribution obtained from the 


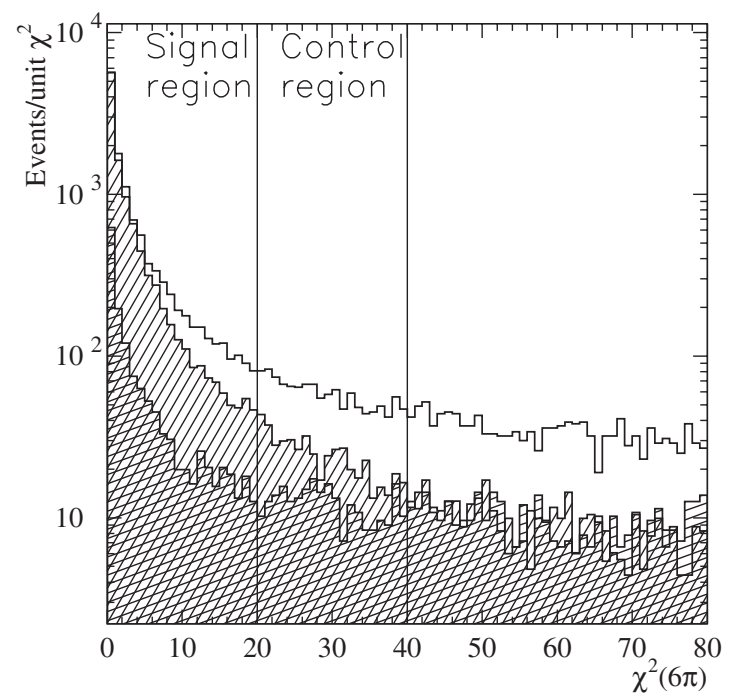

FIG. 1. The one-constraint $\chi^{2}$ distributions for data (unshaded histogram) and MC $3\left(\pi^{+} \pi^{-}\right) \gamma$ simulation (shaded histogram) for six-charged-track events fitted to the six-pion hypothesis. The cross-hatched histogram is the estimated background contribution from non-ISR events obtained from JETSET. The signal and control regions are indicated.

JETSET simulation of quark-antiquark production and hadronization and does not exceed $8 \%$.

We require $\chi_{6 \pi}^{2}<20$ for the six-pion hypothesis, and that any accompanying fit to the $2 K 4 \pi$ hypothesis has $\chi_{2 K 4 \pi}^{2}>20$. The subscripts " $6 \pi$ " and " $2 K 4 \pi$ " here and below refer to the $3\left(\pi^{+} \pi^{-}\right)$and $K^{+} K^{-} 2\left(\pi^{+} \pi^{-}\right)$final states exclusively. We estimate that these requirements reduce the misidentification of $2 K 4 \pi$ events from $11 \%$ to about $2 \%$, at the cost of the loss of about $5 \%$ of the signal $6 \pi$ events.

The region $20<\chi_{6 \pi}^{2}<40$ is chosen as a control region for the estimation of background from other ISR and nonISR multihadron reactions. The procedure followed is described in the next section.

The signal region of Fig. 1 contains 19683 data and $19980 \mathrm{MC}$ events, while for the control region the corresponding numbers are 2021 and 875 , respectively.

\section{B. Background estimation}

The non-ISR background contribution to the signal region is obtained from the JETSET MC simulation, normalized using the integrated $e^{+} e^{-}$luminosity. The $\chi^{2}$ distribution for non-ISR events is shown by the crosshatched histogram of Fig. 1. The non-ISR background dominates by $e^{+} e^{-} \rightarrow 6$ hadrons $+\pi^{0}$ production with a photon from $\pi^{0}$ mistakenly taken as an ISR photon.

MC simulation of the $\tau^{+} \tau^{-}$final state and ISR production of multihadron final states other than $3\left(\pi^{+} \pi^{-}\right)$show that such states yield a background in the selected six-pion sample that exhibits a relatively flat contribution to the $\chi_{6 \pi}^{2}$ distribution. To validate these estimates of backgrounds with the data, we subtract the MC-simulated signal distribution (the shaded histogram of Fig. 1) from the unshaded one, after the non-ISR background is subtracted. The shape of the resulting histogram is well described by MC simulation of remaining background processes. Its absolute normalization is used to estimate the level of those backgrounds in the signal region.

The background contribution to any distribution other than $\chi^{2}$ is estimated as the difference between the distributions in the relevant quantity for data and MC events from the control region of Fig. 1, normalized to the difference between the number of data and MC events in the signal region. The non-ISR background is subtracted separately.

For example, Fig. 2 shows the six-pion invariant-mass distribution up to $4.5 \mathrm{GeV} / \mathrm{c}^{2}$ for the signal region of Fig. 1. The points with error bars show the ISR background contribution obtained in the manner described from the control region of Fig. 1. The cross-hatched histogram in Fig. 2 represents the non-ISR background contribution obtained from the JETSET MC simulation.

Both backgrounds are relatively small at low mass (about 6\%-8\%), but the non-ISR background accounts for about $20 \%-25 \%$ of the observed data at approximately $4 \mathrm{GeV} / \mathrm{c}^{2}$.

Accounting for uncertainties in cross sections for background processes, uncertainties in normalization of events in the control region and statistical fluctuations in the number of simulated events, we estimate that this procedure for background subtraction results in a systematic

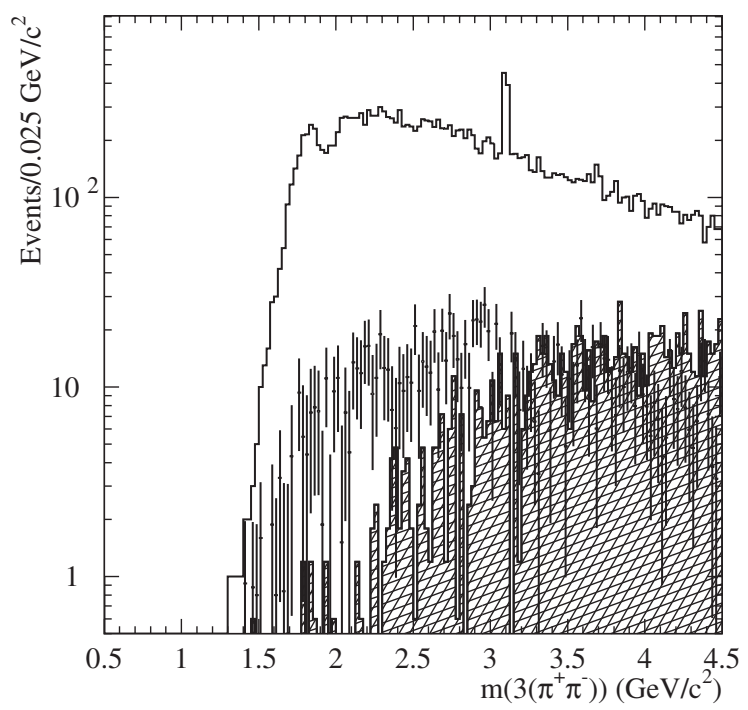

FIG. 2. The six-pion invariant-mass distribution (unshaded histogram) for the signal region of Fig. 1. The points indicate the background estimated from the difference between data and MC events for the control region of Fig. 1, normalized to the difference between data and $\mathrm{MC}$ events in the signal region of Fig. 1. The cross-hatched histogram corresponds to the non-ISR background of Fig. 1. 
THE $e^{+} e^{-} \rightarrow 3\left(\pi^{+} \pi^{-}\right), 2\left(\pi^{+} \pi^{-} \pi^{0}\right)$ AND $K^{+} K^{-} 2\left(\pi^{+} \pi^{-}\right) \ldots$

uncertainty of less than $3 \%$ in the number of signal events in the $1.6-3 \mathrm{GeV} / \mathrm{c}^{2}$ region of six-pion mass, but that it increases to $3 \%-5 \%$ in the region above $3 \mathrm{GeV} / \mathrm{c}^{2}$.

By selecting a "background-free" $6 \pi \gamma$ sample with only six-charged tracks and only one photon (about 5\% of events) we can compare $\chi^{2}$ distributions for data and MC events up to $\chi^{2}=1000$. We estimate that for a $\chi_{6 \pi}^{2}<$ 20 selection the net signal size should be increased by $(3 \pm$ 2)\% to allow for a slight shape difference between the MC and experimental $\chi^{2}$ distributions.

\section{Tracking efficiency}

The procedure to measure the track-finding efficiency is described in our previous paper [8] for the four-pion final state. The method uses events that have three chargedparticle tracks and a hard photon. These events are subjected to a one-constraint fit, which uses all measured parameters of the three tracks and the photon and yields the three-momentum vector of the missing charged pion in the laboratory frame assuming this is the only undetected track. If the $\chi^{2}$ of the fit is less than 30 and this vector lies within the acceptance of the $\mathrm{DCH}$, the event is included in the data sample. The ratio of three- to four-charged-track events gives the track-finding efficiency. The same procedure is applied to MC-simulated events. The track-finding efficiency is better for MC-simulated events by $(0.8 \pm$ 0.5)\% per track independent from momentum [8]. Assuming no increase in correlations due to higher multiplicity, we apply an overall correction of $+(5 \pm 3) \%$ to the observed six-pion event sample based on the previous study.

\section{Detection efficiency from simulation}

The selection procedures applied to the data are also applied to the MC-simulated event sample. The resulting six-pion invariant-mass distribution is shown in Fig. 3(a) for the signal and control (shaded histogram) regions. The mass dependence of the detection efficiency is obtained by dividing the number of reconstructed $\mathrm{MC}$ events in each $25 \mathrm{MeV} / \mathrm{c}^{2}$ mass interval by the number generated in this same interval. Note that the detection efficiency computed that way is insensitive to the actual shape of the mass distribution of Fig. 3(a) used in MC simulation. The result is shown in Fig. 3(b); the curve is obtained from a 3rdorder polynomial fit to the distribution. The efficiency falls off gradually with increasing mass from about $20 \%$ at $1.6 \mathrm{GeV} / \mathrm{c}^{2}$ to about $14 \%$ at $4.5 \mathrm{GeV} / \mathrm{c}^{2}$. This efficiency estimate takes into account the geometrical acceptance of the detector for the final-state photon and the charged pions, the inefficiency of the several detector subsystems, and event loss due to additional soft-photon emission from the initial and final states.

As mentioned in Sec. II, the model used in the MC simulation assumes that the six-pion final state results predominantly from the $\rho(770) 2\left(\pi^{+} \pi^{-}\right)$production pro-
PHYSICAL REVIEW D 73, 052003 (2006)
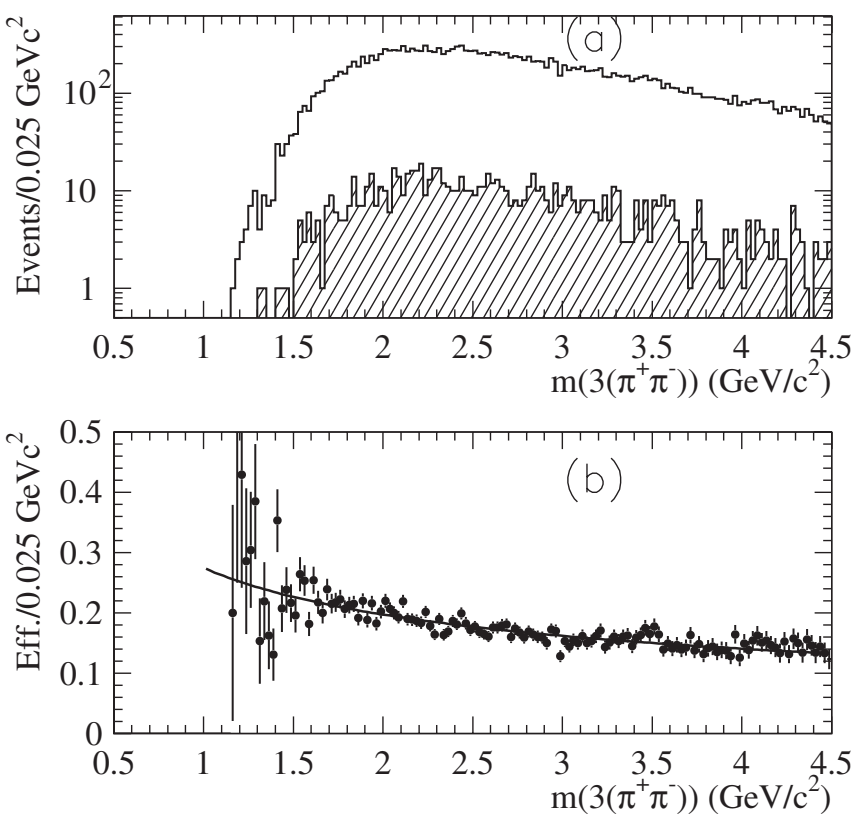

FIG. 3. (a) The six-pion mass distributions from MC simulation for the signal (unshaded) and control (shaded) regions of Fig. 1. (b) The mass dependence of the net reconstruction and selection efficiency obtained from simulation. The curve is a fit described in the text.

cess. In general, this model describes well the distributions in many of the kinematic variables characterizing the sixpion final state. Some examples are shown in Figs. 4 and 5, in which the points with error bars represent data while the histograms are obtained from MC simulation. Figure 4(a) shows the distribution in $\psi_{\min }$, the minimum charged-pionpair opening angle for each event, while Figs. 4(b) and 4(c) represent the distribution in polar angle, $\theta_{\mathrm{ch}}$, and transverse momentum, $p_{T}$, respectively, for all final-state pions. All quantities are calculated in the laboratory frame. The overall agreement between MC simulation and data is very good. Figure 5 compares the distributions in $\cos \theta$, where
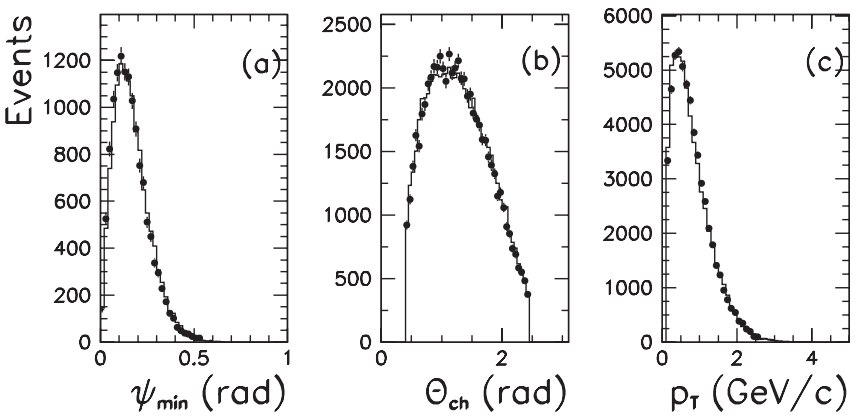

FIG. 4. (a) The distribution in the track-pair opening angle for the minimum of the 15 values possible for each event; (b) the distribution in polar angle; (c) the transverse momentum distribution for all pions from all events. All quantities are in the laboratory frame; the points are for data and the histograms are obtained from MC simulation. 

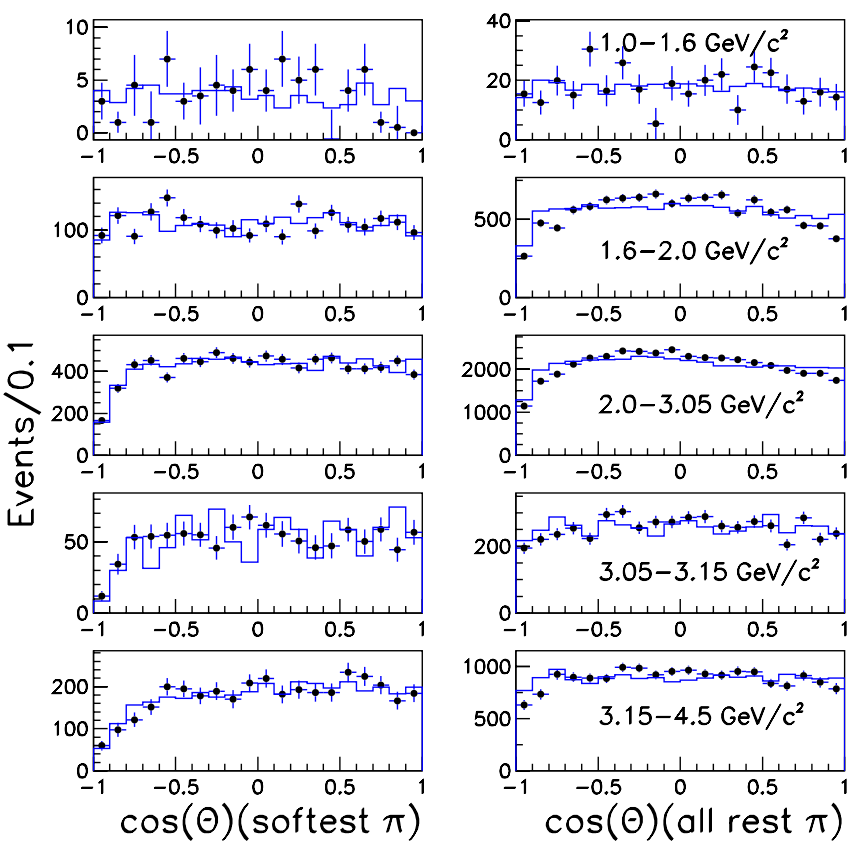

FIG. 5 (color online). The angular distribution of the lowestmomentum pion (left) and of the sum of the remaining five most energetic pions (right) in the six-pion rest frame with respect to the direction of the six-pion system in the laboratory frame for the five regions of six-pion mass indicated in the right-hand plots. The fourth slice is chosen to correspond to the $J / \psi$ region. The points are data, and the histograms are MC simulation.

$\theta$ is the angle between a charged pion in the six-pion rest frame, and the direction of the six-pion system in the laboratory frame. Data and $\mathrm{MC}$ are in rather good agreement.

In the six-pion rest frame, the angular acceptance is rather uniform. A simulation without resonances using only six-pion phase space does not produce discernible deviations from the observed angular distributions, and does not change the overall acceptance by more than $3 \%$. This value is taken as an estimate of the model-dependent systematic uncertainty in the acceptance.

\section{E. Cross section for $e^{+} e^{-} \rightarrow 3\left(\pi^{+} \pi^{-}\right)$}

Data from the reaction $e^{+} e^{-} \rightarrow \mu^{+} \mu^{-} \gamma$ are used to convert the invariant-mass distribution for an ISRproduced hadronic final state to the energy dependence of the corresponding $e^{+} e^{-}$cross section. The invariant mass of the muon pair $m_{\text {inv }}^{\mu \mu}$ defines an effective $e^{+} e^{-}$c.m. collision energy, $E_{\text {c.m. }}$. The differential luminosity, $d \mathcal{L}$, associated with the interval $d E_{\mathrm{c} . \mathrm{m}}$. centered at effective collision energy $E_{\text {c.m. }}$ is then obtained from

$d \mathcal{L}\left(E_{\mathrm{c} . \mathrm{m} .}\right)=\frac{d N_{\mu \mu \gamma}\left(E_{\mathrm{c} . \mathrm{m} .}\right)}{\epsilon_{\mu \mu} \cdot\left(1+\delta_{\mathrm{FSR}}^{\mu \mu}\right) \cdot \sigma_{\mu^{+} \mu^{-}}\left(E_{\mathrm{c} . \mathrm{m} .}\right) \cdot\left(1+\delta_{\mathrm{vac}}\right)}$,

where $E_{\text {c.m. }} \equiv m_{\mathrm{inv}}^{\mu \mu} ; d N_{\mu \mu \gamma}$ is the number of muon pairs in the mass interval $d m_{\text {inv }}^{\mu \mu} \equiv d E_{\text {c.m. }} ; \epsilon_{\mu \mu}$ is the acceptance, corrected for muon identification and soft-photon emission; $\left(1+\delta_{\mathrm{FSR}}^{\mu \mu}\right)$ corrects for hard photon emission from final-state muons; $\sigma_{\mu^{+} \mu^{-}}\left(E_{\mathrm{c} . \mathrm{m} .}\right)$ is the $e^{+} e^{-} \rightarrow \mu^{+} \mu^{-}$ Born cross section at center-of-mass energy $E_{\text {c.m. }}$; and $(1+$ $\left.\delta_{\text {vac }}\right)$ is the corresponding vacuum polarization correction [17]. The dependence of the differential luminosity on $E_{\text {c.m. }}$ is presented in our previous paper [8].

From a detailed study of the $e^{+} e^{-} \rightarrow \mu^{+} \mu^{-} \gamma$ detection and identification efficiency described in Ref. [6] and comparison of the observed invariant-mass spectrum with theoretical calculations, we estimate the systematic uncertainty associated with luminosity determination to be $3 \%$.

The six-pion $e^{+} e^{-}$cross section can then be calculated from

$$
\sigma\left(3\left(\pi^{+} \pi^{-}\right)\right)\left(E_{\mathrm{c} . \mathrm{m} .}\right)=\frac{d N_{6 \pi \gamma}\left(E_{\mathrm{c} . \mathrm{m} .}\right)}{d \mathcal{L}\left(E_{\mathrm{c} . \mathrm{m} .}\right) \cdot \epsilon_{6 \pi}^{\mathrm{corr}} \cdot \epsilon_{6 \pi}^{\mathrm{MC}}\left(E_{\mathrm{c} . \mathrm{m} .}\right)},
$$

where $m_{\mathrm{inv}}^{6 \pi} \equiv E_{\text {c.m. }}$. with $m_{\mathrm{inv}}^{6 \pi}$ the invariant mass of the sixcharged-pion system; $d N_{6 \pi \gamma}$ is the number of selected sixcharged-pion events after background subtraction in the interval $d E_{\text {c.m. }}$ and $\epsilon_{6 \pi}^{\mathrm{MC}}\left(E_{\text {c.m. }}\right)$ is the corresponding detection efficiency obtained from the MC simulation. The factor $\epsilon_{6 \pi}^{\text {corr }}$ takes into account the difference between the $\chi^{2}$ distributions for data and $\mathrm{MC}$ events, and the trackingefficiency discrepancies discussed in Sec. IV B and IV C respectively.

The energy dependence of the cross section for the reaction $e^{+} e^{-} \rightarrow 3\left(\pi^{+} \pi^{-}\right)$after all corrections is shown in Fig. 6. It shows a structure around $1.9 \mathrm{GeV}$ and reaches a

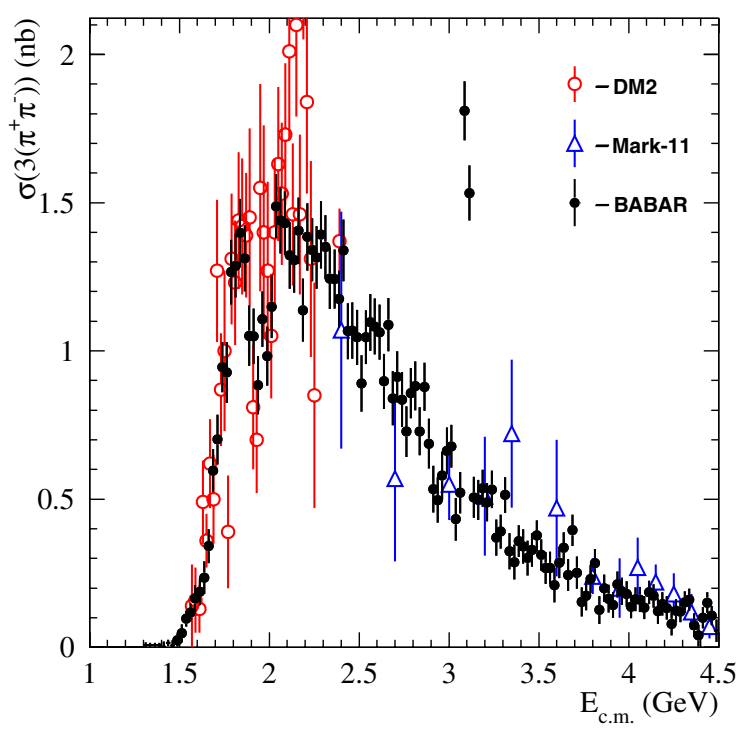

FIG. 6 (color online). The $e^{+} e^{-}$c.m. energy dependence of the $3\left(\pi^{+} \pi^{-}\right)$cross section measured with ISR data at BABAR compared with the direct $e^{+} e^{-}$measurements by DM2 and MARK-II. Only statistical errors are shown. 
THE $e^{+} e^{-} \rightarrow 3\left(\pi^{+} \pi^{-}\right), 2\left(\pi^{+} \pi^{-} \pi^{0}\right)$ AND $K^{+} K^{-} 2\left(\pi^{+} \pi^{-}\right) \ldots$

PHYSICAL REVIEW D 73, 052003 (2006)

TABLE I. Summary of the $e^{+} e^{-} \rightarrow 3\left(\pi^{+} \pi^{-}\right)$cross section measurement. Errors are statistical only.

\begin{tabular}{|c|c|c|c|c|c|c|c|}
\hline$E_{\text {c.m. }}(\mathrm{GeV})$ & $\sigma(\mathrm{nb})$ & $E_{\text {c.m. }}(\mathrm{GeV})$ & $\sigma(\mathrm{nb})$ & $E_{\text {c.m. }}(\mathrm{GeV})$ & $\sigma(\mathrm{nb})$ & $E_{\text {c.m. }}(\mathrm{GeV})$ & $\sigma(\mathrm{nb})$ \\
\hline 1.3125 & $0.01 \pm 0.01$ & 2.1125 & $1.36 \pm 0.12$ & 2.9125 & $0.55 \pm 0.08$ & 3.7125 & $0.26 \pm 0.06$ \\
\hline 1.3375 & $0.01 \pm 0.01$ & 2.1375 & $1.35 \pm 0.11$ & 2.9375 & $0.51 \pm 0.08$ & 3.7375 & $0.16 \pm 0.05$ \\
\hline 1.3625 & $0.01 \pm 0.01$ & 2.1625 & $1.45 \pm 0.12$ & 2.9625 & $0.60 \pm 0.08$ & 3.7625 & $0.18 \pm 0.05$ \\
\hline 1.3875 & $0.01 \pm 0.01$ & 2.1875 & $1.17 \pm 0.11$ & 2.9875 & $0.68 \pm 0.08$ & 3.7875 & $0.24 \pm 0.05$ \\
\hline 1.4125 & $0.00 \pm 0.02$ & 2.2125 & $1.43 \pm 0.12$ & 3.0125 & $0.70 \pm 0.07$ & 3.8125 & $0.29 \pm 0.05$ \\
\hline 1.4375 & $0.02 \pm 0.01$ & 2.2375 & $1.38 \pm 0.11$ & 3.0375 & $0.45 \pm 0.07$ & 3.8375 & $0.13 \pm 0.05$ \\
\hline 1.4625 & $0.01 \pm 0.02$ & 2.2625 & $1.36 \pm 0.11$ & 3.0625 & $0.54 \pm 0.07$ & 3.8625 & $0.21 \pm 0.05$ \\
\hline 1.4875 & $0.03 \pm 0.02$ & 2.2875 & $1.44 \pm 0.12$ & 3.0875 & $1.87 \pm 0.10$ & 3.8875 & $0.17 \pm 0.05$ \\
\hline 1.5125 & $0.05 \pm 0.03$ & 2.3125 & $1.40 \pm 0.11$ & 3.1125 & $1.58 \pm 0.10$ & 3.9125 & $0.15 \pm 0.04$ \\
\hline 1.5375 & $0.10 \pm 0.03$ & 2.3375 & $1.28 \pm 0.11$ & 3.1375 & $0.52 \pm 0.07$ & 3.9375 & $0.22 \pm 0.05$ \\
\hline 1.5625 & $0.12 \pm 0.03$ & 2.3625 & $1.28 \pm 0.10$ & 3.1625 & $0.51 \pm 0.07$ & 3.9625 & $0.20 \pm 0.05$ \\
\hline 1.5875 & $0.17 \pm 0.05$ & 2.3875 & $1.21 \pm 0.10$ & 3.1875 & $0.55 \pm 0.06$ & 3.9875 & $0.19 \pm 0.04$ \\
\hline 1.6125 & $0.19 \pm 0.05$ & 2.4125 & $1.38 \pm 0.11$ & 3.2125 & $0.51 \pm 0.07$ & 4.0125 & $0.14 \pm 0.04$ \\
\hline 1.6375 & $0.24 \pm 0.06$ & 2.4375 & $1.10 \pm 0.10$ & 3.2375 & $0.55 \pm 0.07$ & 4.0375 & $0.17 \pm 0.04$ \\
\hline 1.6625 & $0.35 \pm 0.06$ & 2.4625 & $1.10 \pm 0.10$ & 3.2625 & $0.38 \pm 0.06$ & 4.0625 & $0.17 \pm 0.04$ \\
\hline 1.6875 & $0.62 \pm 0.07$ & 2.4875 & $1.08 \pm 0.10$ & 3.2875 & $0.40 \pm 0.06$ & 4.0875 & $0.14 \pm 0.04$ \\
\hline 1.7125 & $0.72 \pm 0.09$ & 2.5125 & $0.92 \pm 0.10$ & 3.3125 & $0.53 \pm 0.06$ & 4.1125 & $0.19 \pm 0.04$ \\
\hline 1.7375 & $0.98 \pm 0.09$ & 2.5375 & $1.08 \pm 0.09$ & 3.3375 & $0.33 \pm 0.06$ & 4.1375 & $0.18 \pm 0.04$ \\
\hline 1.7625 & $0.96 \pm 0.11$ & 2.5625 & $1.13 \pm 0.10$ & 3.3625 & $0.30 \pm 0.06$ & 4.1625 & $0.13 \pm 0.04$ \\
\hline 1.7875 & $1.31 \pm 0.11$ & 2.5875 & $1.12 \pm 0.10$ & 3.3875 & $0.37 \pm 0.06$ & 4.1875 & $0.15 \pm 0.04$ \\
\hline 1.8125 & $1.33 \pm 0.11$ & 2.6125 & $1.10 \pm 0.10$ & 3.4125 & $0.35 \pm 0.06$ & 4.2125 & $0.14 \pm 0.04$ \\
\hline 1.8375 & $1.44 \pm 0.12$ & 2.6375 & $0.93 \pm 0.10$ & 3.4375 & $0.31 \pm 0.06$ & 4.2375 & $0.08 \pm 0.04$ \\
\hline 1.8625 & $1.35 \pm 0.12$ & 2.6625 & $1.12 \pm 0.09$ & 3.4625 & $0.34 \pm 0.06$ & 4.2625 & $0.13 \pm 0.04$ \\
\hline 1.8875 & $1.09 \pm 0.11$ & 2.6875 & $0.87 \pm 0.09$ & 3.4875 & $0.39 \pm 0.05$ & 4.2875 & $0.13 \pm 0.04$ \\
\hline 1.9125 & $1.08 \pm 0.10$ & 2.7125 & $0.94 \pm 0.09$ & 3.5125 & $0.32 \pm 0.05$ & 4.3125 & $0.16 \pm 0.04$ \\
\hline 1.9375 & $0.91 \pm 0.10$ & 2.7375 & $0.86 \pm 0.10$ & 3.5375 & $0.28 \pm 0.05$ & 4.3375 & $0.17 \pm 0.04$ \\
\hline 1.9625 & $1.14 \pm 0.10$ & 2.7625 & $0.75 \pm 0.09$ & 3.5625 & $0.28 \pm 0.06$ & 4.3625 & $0.08 \pm 0.04$ \\
\hline 1.9875 & $1.01 \pm 0.10$ & 2.7875 & $0.89 \pm 0.09$ & 3.5875 & $0.22 \pm 0.06$ & 4.3875 & $0.04 \pm 0.04$ \\
\hline 2.0125 & $1.19 \pm 0.11$ & 2.8125 & $0.91 \pm 0.09$ & 3.6125 & $0.30 \pm 0.05$ & 4.4125 & $0.10 \pm 0.04$ \\
\hline 2.0375 & $1.54 \pm 0.11$ & 2.8375 & $0.75 \pm 0.08$ & 3.6375 & $0.35 \pm 0.05$ & 4.4375 & $0.16 \pm 0.04$ \\
\hline 2.0625 & $1.49 \pm 0.11$ & 2.8625 & $0.91 \pm 0.08$ & 3.6625 & $0.25 \pm 0.05$ & 4.4625 & $0.11 \pm 0.03$ \\
\hline 2.0875 & $1.48 \pm 0.11$ & 2.8875 & $0.71 \pm 0.09$ & 3.6875 & $0.41 \pm 0.05$ & 4.4875 & $0.06 \pm 0.04$ \\
\hline
\end{tabular}

peak value of about $1.5 \mathrm{nb}$ near $2.0 \mathrm{GeV}$, followed by a monotonic decrease toward higher energies perturbed only by a peak at the $J / \psi$ mass position. The cross section for each $25 \mathrm{MeV}$ interval is presented in Table I.

Since $d \mathcal{L}$ has been corrected for vacuum polarization and final-state soft-photon emission, the six-pion cross section measured in this way includes effects due to vacuum polarization and final-state soft-photon emission. For $g-2$ calculations, vacuum polarization contributions should be excluded from this data.

We studied the resolution in six-pion mass with $\mathrm{MC}$ simulation, finding that Gaussian fits of line shapes give

TABLE II. Summary of systematic errors for the $e^{+} e^{-} \rightarrow 3\left(\pi^{+} \pi^{-}\right)$cross section measurement.

\begin{tabular}{lcc}
\hline \hline Source & Correction applied & Systematic error \\
\hline Luminosity from $\mu \mu \gamma$ & $\ldots$ & $3 \%$ \\
MC-data difference in $\chi^{2}<20$ signal region & $+3 \%$ & $2 \%$ \\
Background subtraction & $\cdots$ & $3 \%$ for $m_{6 \pi}<3.0 \mathrm{GeV} / \mathrm{c}^{2}$ \\
& & $5 \%$ for $m_{6 \pi}>3.0 \mathrm{GeV} / \mathrm{c}^{2}$ \\
MC-data difference in tracking efficiency & $+5 \%$ & $3 \%$ \\
Radiative corrections accuracy & $\cdots$ & $1 \%$ \\
Acceptance from MC (model-dependent) & $\cdots$ & $3 \%$ \\
Total (assuming addition in quadrature and no correlations) & $+8 \%$ & $6 \%$ for $m_{6 \pi}<3.0 \mathrm{GeV} / \mathrm{c}^{2}$ \\
& & $8 \%$ for $m_{6 \pi}>3.0 \mathrm{GeV} / \mathrm{c}^{2}$ \\
\hline \hline
\end{tabular}


mass resolutions $\sigma_{\text {res }}$ that vary between $6.2 \mathrm{MeV} / \mathrm{c}^{2}$ in the $1.5-2.5 \mathrm{GeV} / \mathrm{c}^{2}$ mass region and $8.7 \mathrm{MeV} / \mathrm{c}^{2}$ in the $2.5-3.5 \mathrm{GeV} / \mathrm{c}^{2}$ mass region. The observed line shape is not purely Gaussian mainly due to soft-photon radiation. Since the resolution varies slowly with mass, the data are binned in mass intervals of $25 \mathrm{MeV} / \mathrm{c}^{2}$ and the cross section has no sharp peaks (except for the $J / \psi$ region discussed below), unfolding the resolution has little effect on the measured energy dependence and has not been performed in this analysis. For the sake of any future comparisons of our data with models (e.g. for the structure near $1.9 \mathrm{GeV} / \mathrm{c}^{2}$ ) we provide the relation between the observed number $N_{i}$ of events in bin $i$ and the "true" number of events in nearby bins:

$$
\begin{aligned}
N_{i}= & e 1 \cdot N_{i-2}^{0}+e 2 \cdot N_{i-1}^{0}+e 3 \cdot N_{i}^{0}+e 4 \cdot N_{i+1}^{0} \\
& +e 5 \cdot N_{i+2}^{0},
\end{aligned}
$$

where $N_{i}^{0}$ is the number of events in bin $i$ before accounting for resolution. The coefficients $e 1, \ldots, e 5=0.005,0.031$, $0.896,0.062,0.006$ are the true event fractions in five energy bins of $25 \mathrm{MeV}$ width for the six-pion mass region $1.5-2.5 \mathrm{GeV} / \mathrm{c}^{2}$.

\section{F. Summary of systematic studies}

The measured six-charged-pion cross section values shown in Fig. 6 and summarized in Table I include only statistical errors. The systematic errors discussed in previous sections are summarized in Table II, along with the corrections that were applied to the measurements.

The two systematic corrections applied to the measured cross sections sum up to $+8 \%$ with $6 \%-8 \%$ taken as a systematic uncertainty.

\section{G. Physics results}

The six-charged-pion cross section measured by $B A B A R$ can be compared with existing $e^{+} e^{-}$measurements performed by the DM2 [18] and MARK-II [19] detectors (see Fig. 6). The agreement is relatively good, but the $B A B A R$ measurement is much more precise. The structure around $1.9 \mathrm{GeV}$ reported by both the DM2 [18] and FOCUS [20] experiments is clearly seen (see discussion later).

Different mass combinations were studied in data and MC events to search for any structures or states not included in the simulation. Figure 7 shows the scatter plots of $4 \pi$ and $2 \pi$ mass versus $6 \pi$ mass for data and MC events. Good agreement is seen except for narrow regions around the $J / \psi$ and $\psi(2 S)$ masses that are not included in the simulation.

In order to make a more detailed study, five intervals of $6 \pi$ mass are selected: (1) $1.0-1.6 \mathrm{GeV} / \mathrm{c}^{2}$; (2) $1.6-2.0 \mathrm{GeV} / \mathrm{c}^{2}$ (an interval with significant structure in the cross section, see Fig. 6); (3) $2.0-3.05 \mathrm{GeV} / \mathrm{c}^{2}$; (4) $3.05-3.15 \mathrm{GeV} / \mathrm{c}^{2}$ (the $J / \psi$ resonance region); and (5) $3.0-4.5 \mathrm{GeV} / \mathrm{c}^{2}$. Figure 8 shows the two- and three-
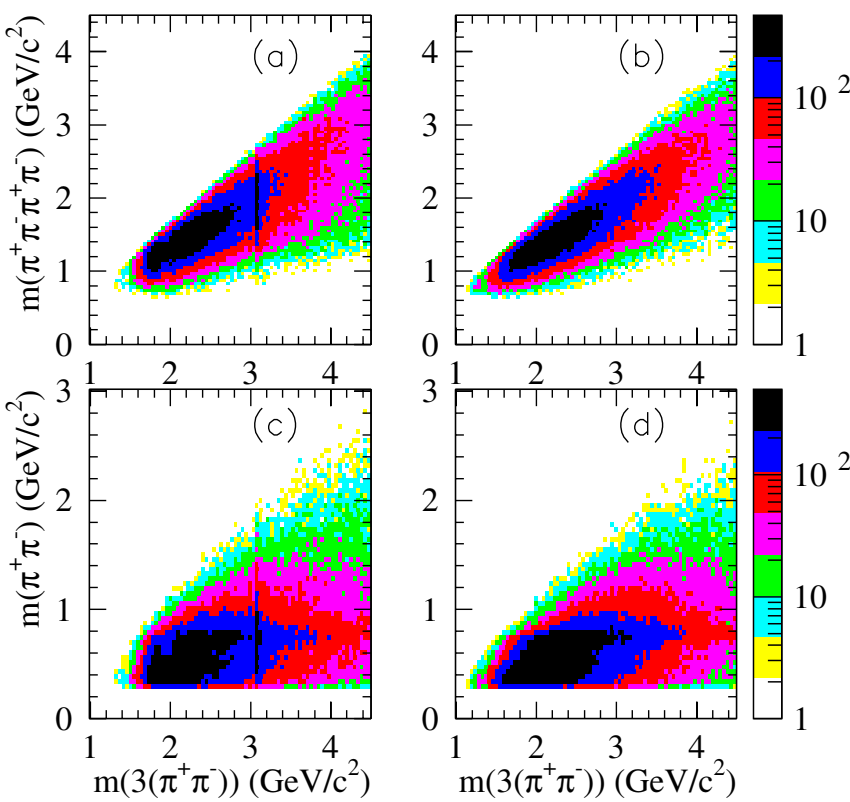

FIG. 7 (color online). Invariant-mass distributions for all sixpion versus neutral four-pion combinations (top) and neutral two-pion combinations (bottom) for data [(a), (c)] and MC simulation [(b), (d)]. The $J / \psi$ signal seen in the data is not included in the simulation.

pion mass projections, as well as the mass projections of the remaining four pions in events having a two-pion combination within $\pm 75 \mathrm{MeV} / \mathrm{c}^{2}$ of the $\rho$ mass, for the five regions of six-pion mass just described, for both data and simulation. Background in the data is subtracted using the $\chi^{2}$ distributions as described above.

A simple model with only one $\rho(770)$ per event is in excellent agreement with experimental data. No other significant structures are observed. A full partial wave analysis (PWA) would be required in order to arrive at a more precise interpretation of the data but the final state with only one $\rho(770)$ per event dominates. This PWA requires a simultaneous analysis of the $2\left(\pi^{+} \pi^{-} \pi^{0}\right)$ final state, which is described in the next sections.

\section{THE $2\left(\pi^{+} \pi^{-} \pi^{0}\right)$ FINAL STATE}

\section{A. Additional selection criteria}

The results of the five-constraint fit (see Sec. III) to the four-charged-track and five-photon candidates are used to make the final selection of the $2\left(\pi^{+} \pi^{-} \pi^{0}\right)$ sample. The polar angle $\theta_{\mathrm{ch}}^{\text {fit }}$ of each charged track obtained from the fit has to satisfy $0.45<\theta_{\mathrm{ch}}^{\mathrm{fit}}<2.4 \mathrm{rad}$. To reduce the background from events where an energetic photon is coupled with a soft photon from background to form a $\pi^{0}$, an additional selection is applied. The cosine of the helicity angle of the photons of the most energetic $\pi^{0}$, measured in the $\pi^{0}$ rest frame, is required to be less than 0.85 . We further require all photons from $\pi^{0}$ to have an energy higher than $50 \mathrm{MeV}$. 

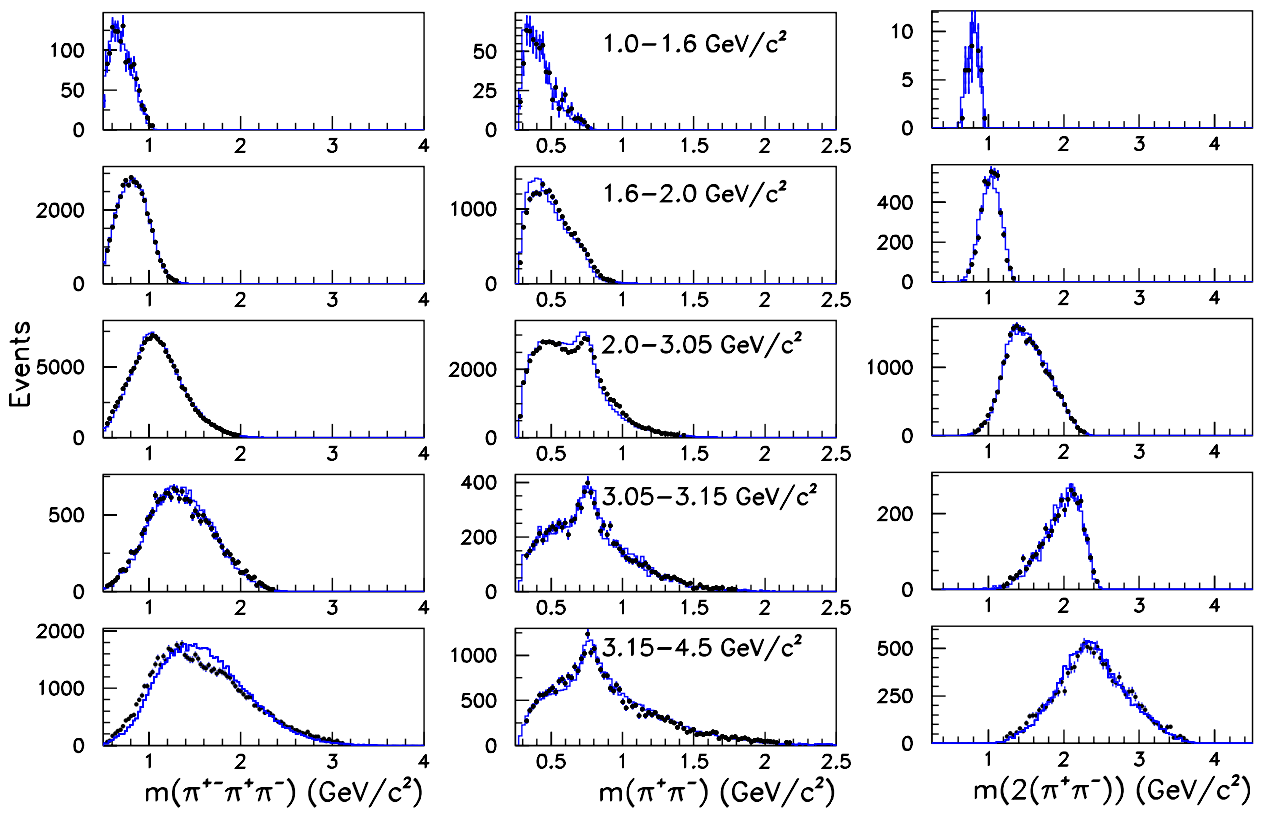

FIG. 8 (color online). Invariant-mass distributions for different subcombinations of pions arranged by rows for the five different regions of $3\left(\pi^{+} \pi^{-}\right)$mass indicated in the central column of histograms. The points (histograms) display data (simulation). The threepion (left column) and two-pion (middle column) plots sum over all possible combinations, while the $2\left(\pi^{+} \pi^{-}\right.$) combinations (right column) include only the remaining combinations from events selected to have one $\pi^{+} \pi^{-}$in the $\rho(770)$ region.

After these additional selections we require $\chi_{4 \pi 2 \pi^{0}}^{2}<40$ for the six-pion hypothesis and require that none of the charged tracks be identified as a kaon to reduce the contribution from $K^{+} K^{-} \pi^{+} \pi^{-} \pi^{0} \pi^{0}$ and $K K_{S} 3 \pi$ final states.

The five-constraint-fit $\chi^{2}$ distribution for the four-pion and five-photon candidates is shown as the unshaded histogram of Fig. 9, while the shaded region is for the corresponding MC-simulated pure $2\left(\pi^{+} \pi^{-} \pi^{0}\right) \gamma$ events. The MC-simulated $\chi^{2}$ distribution is normalized to the data in the region $\chi^{2}<10$ where contamination of the background events and multiple soft ISR and FSR is lowest.

The cross-hatched histogram in Fig. 9 represents the non-ISR background contribution obtained from the JETSET simulation of quark-antiquark production and hadronization and does not exceed $10 \%$.

The region $40<\chi_{4 \pi 2 \pi^{0}}^{2}<80$ is chosen as a control region for the estimation of background from other ISR and non-ISR multihadron reactions.

The signal region of Fig. 9 contains 35499 data and 6833 MC events, while for the control region the corresponding numbers are 8421 and 672, respectively.

\section{B. Background estimation}

The background subtraction procedure for the $2\left(\pi^{+} \pi^{-} \pi^{0}\right)$ final state is identical to that already described in Sec. IV B for the $3\left(\pi^{+} \pi^{-}\right)$final state, using the $\chi_{4 \pi 2 \pi^{0}}^{2}$ distributions shown in Fig. 9. The unshaded histogram of Fig. 10 shows the $2\left(\pi^{+} \pi^{-} \pi^{0}\right)$ invariant-mass distribution for the signal region of Fig. 9. The points with error bars show the ISR background contribution obtained in the manner described from the control region of Fig. 9. The cross-hatched histogram in Fig. 10 represents the non-ISR background contribution obtained from the JETSET MC simulation. Both backgrounds are relatively small at low mass (about 10\%), but the non-ISR background accounts

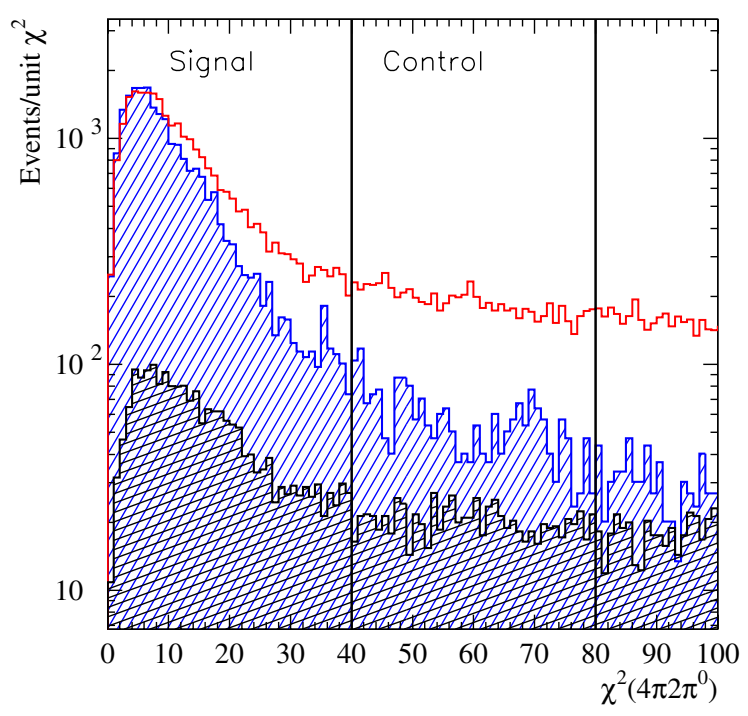

FIG. 9 (color online). The five-constraint $\chi^{2}$ distributions for data (unshaded histogram) and MC $2\left(\pi^{+} \pi^{-} \pi^{0}\right) \gamma$ simulation (shaded) for four-charged-track and five-photon events fitted to the six-pion hypothesis. The cross-hatched histogram is the estimated background contribution from non-ISR events obtained from JETSET. The signal and control regions are indicated. 


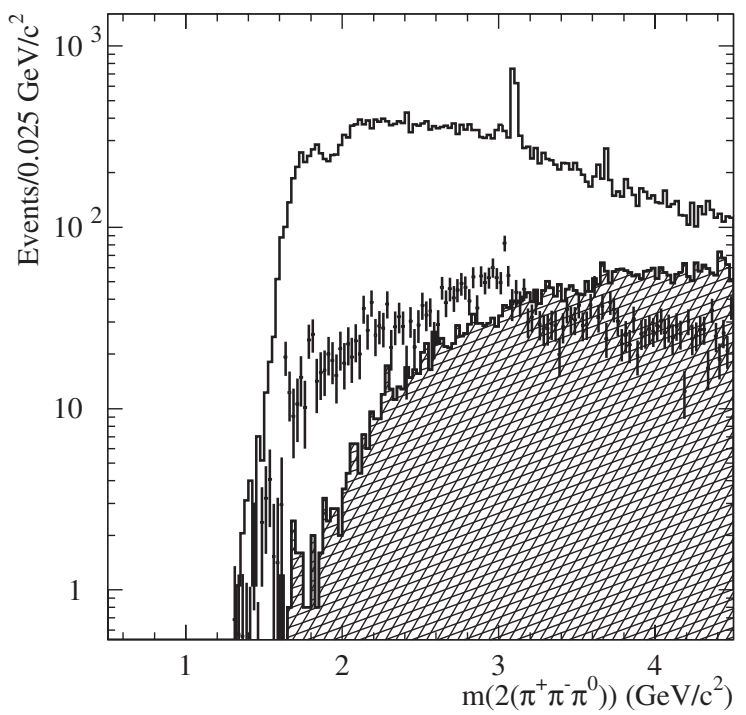

FIG. 10. The six-pion (four-charged and two neutral) invariant-mass distribution (unshaded histogram) for the signal region of Fig. 9. The points indicate the background estimated from the difference between data and MC events for the control region of Fig. 9, normalized to the difference between data and MC events in the signal region of Fig. 9. The cross-hatched histogram corresponds to the non-ISR background of Fig. 9.

for about $20 \%-25 \%$ of the observed data at approximately $4 \mathrm{GeV} / \mathrm{c}^{2}$.

Accounting for uncertainties in cross sections for background processes, uncertainties in normalization of the control sample and statistical fluctuations in the number of simulated events, we estimate that this procedure for background subtraction results in a systematic uncertainty of less than $3 \%$ in the number of signal events in the $1.6-3 \mathrm{GeV} / \mathrm{c}^{2}$ region of six-pion mass, but that the uncertainty increases to $5 \%-10 \%$ in the region above $3 \mathrm{GeV} / \mathrm{c}^{2}$.

By selecting a "background-free" $2\left(\pi^{+} \pi^{-} \pi^{0}\right) \gamma$ sample with only four-charged tracks and only five photons (about $5 \%$ of events) we find that $\chi^{2}$ distributions for data and MC have similar shapes. The ratio of MC events selected by $\chi_{4 \pi 2 \pi^{0}}^{2}<1000$ and $\chi_{4 \pi 2 \pi^{0}}^{2}<40$ cuts is 1.14 reflecting softphoton radiation processes. An estimate for this ratio can also be made directly from the data by measuring the relative number of $J / \psi$ events over continuum for the $\chi^{2}$ regions noted, yielding the ratio $1.08 \pm 0.04$, in good agreement with the estimate from simulation. The $6 \%$ difference between the two estimates is taken as the estimate of systematic error for the $\chi_{4 \pi 2 \pi^{0}}^{2}<40$ selection.

\section{Pion-finding efficiency}

The charged-pion tracking inefficiency is corrected by applying a $+(3 \pm 2) \%$ correction to the number of observed $2\left(\pi^{+} \pi^{-} \pi^{0}\right)$ ISR events, following the prescription discussed above in Sec. IV C.

The difference in the $\pi^{0}$-finding efficiencies between data and MC events has been studied previously using the $\pi^{+} \pi^{-} \pi^{0} \gamma$ reaction [7]. To extend this study to the case where there are two $\pi^{0}$ 's in the final state, a high statistics sample of ISR-produced $\omega \pi^{0} \rightarrow \pi^{+} \pi^{-} \pi^{0} \pi^{0}$ events is selected using a $1 \mathrm{C}$ fit that ignores the $\pi^{0}$ from the $\omega$ decay. The $\pi^{0}$-finding efficiency is then computed by comparing the number of events in the $\omega$ peak where the $\pi^{0}$ is found to the number of events where it is not. By comparing data and $\mathrm{MC}$ results, it is found that the $\pi^{0}$ efficiency for simulation is $2.8 \pm 1.0 \pm 1.0 \%$ higher than data, where the systematic error comes mostly from the background subtraction procedure. Assuming no correlation in $\pi^{0}$-finding efficiency for two $\pi^{0}$ 's in the event we apply a $+5.6 \%$ overall correction to which we assign a systematic error of $3 \%$.

\section{Detection efficiency from simulation}

The detection efficiency is determined in the same manner described in Sec. IV D. The simulated $2\left(\pi^{+} \pi^{-} \pi^{0}\right)$ invariant-mass distributions after selection are shown in Fig. 11(a) for the signal and control (shaded histogram) regions. The mass dependence of the detection efficiency is obtained by dividing the number of reconstructed $\mathrm{MC}$ events in each $25 \mathrm{MeV} / \mathrm{c}^{2}$ mass interval by the number generated in this same interval. The result is shown in Fig. 11(b) and demonstrates practically uniform efficiency versus mass. This efficiency estimate takes into account the geometrical acceptance of the detector for the final-state photon and the charged and neutral pions, the inefficiency
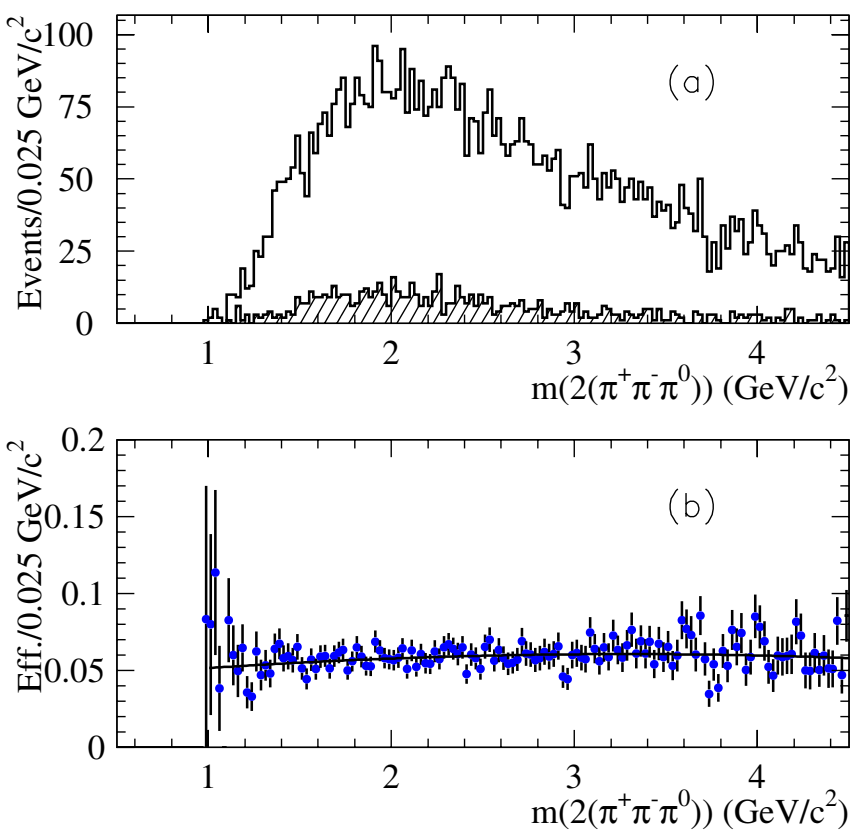

FIG. 11 (color online). (a) The six-pion (four-charged and two neutral) mass distribution from MC simulation for the signal and control (shaded) regions of Fig. 9. (b) The mass dependence of the net reconstruction and selection efficiency obtained from simulation. 
of several detector subsystems, and event loss due to additional soft-photon emission from the initial and final states.

As mentioned in Sec. II, the model used in the MC simulation for $2\left(\pi^{+} \pi^{-} \pi^{0}\right)$ is pure phase space. In general, this model describes the distributions of all the kinematic variables characterizing the final state well, as demonstrated in Fig. 12. The uniform angular acceptance in the rest frame of the final-state pions makes the detection efficiency quite insensitive to the presence of intermediate resonance structures. This feature has also been demonstrated in the six-charged-pion analysis discussed above and in our earlier study of four-charged pions [8], where MC simulations either with intermediate resonances or with phase space differ by no more than $3 \%$ in detection efficiency. We include this $3 \%$ here as a conservative estimate of the systematic error for the model dependence of the $2\left(\pi^{+} \pi^{-} \pi^{0}\right)$ detection efficiency.

\section{E. Cross section for $e^{+} e^{-} \rightarrow \mathbf{2}\left(\pi^{+} \pi^{-} \pi^{0}\right)$}

The $e^{+} e^{-}$energy-dependent cross section for the $2\left(\pi^{+} \pi^{-} \pi^{0}\right)$ final state can then be calculated from

$$
\sigma\left(4 \pi 2 \pi^{0}\right)\left(E_{\mathrm{c} . \mathrm{m} .}\right)=\frac{d N_{4 \pi 2 \pi^{0} \gamma}\left(E_{\mathrm{c.m} .}\right)}{d \mathcal{L}\left(E_{\mathrm{c.m} .}\right) \cdot \epsilon_{4 \pi 2 \pi^{0}}^{\mathrm{corr}} \cdot \epsilon_{4 \pi 2 \pi^{0}}^{\mathrm{MC}}\left(E_{\mathrm{c} . \mathrm{m} .}\right)},
$$
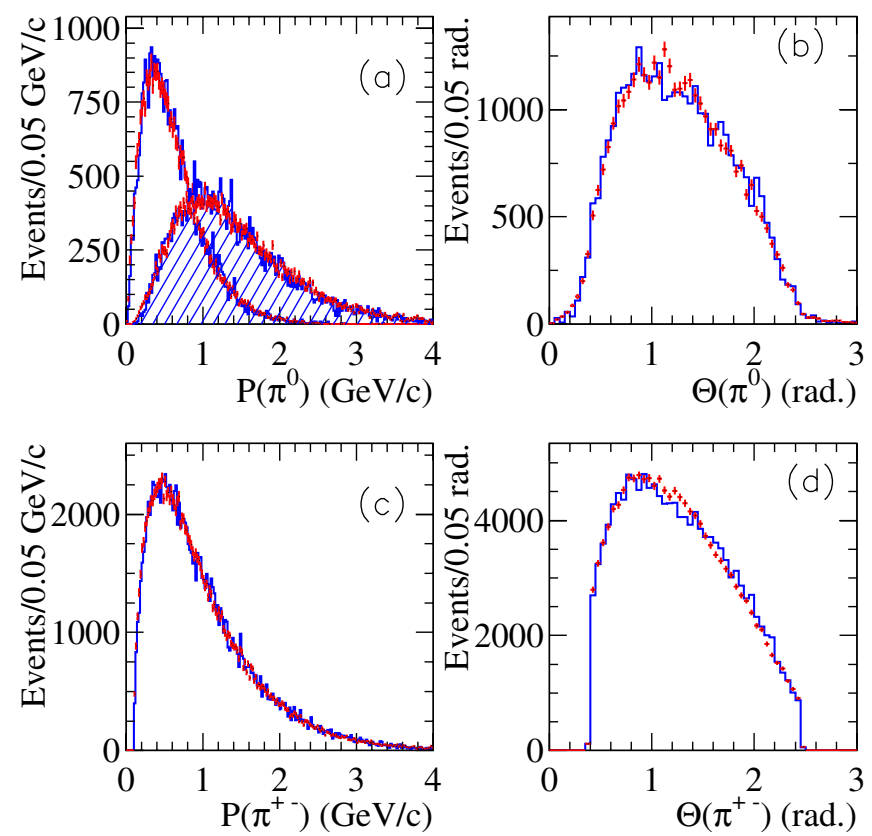

FIG. 12 (color online). Comparison of different kinematic parameters for $2\left(\pi^{+} \pi^{-} \pi^{0}\right)$ events for data (points) and MC simulation (histograms): (a) momentum distributions for the fast (shaded) and slow (unshaded) $\pi^{0}$ 's from MC compared with the data; (b) combined polar angle distribution for the $\pi^{0}$ 's; (c) combined momentum distribution for the two-charged pions; (d) combined polar angle distribution for the two-charged pions. where $m_{\text {inv }}^{4 \pi 2 \pi^{0}} \equiv E_{\text {c.m. }}$, with $m_{\text {inv }}^{4 \pi 2 \pi^{0}}$ the invariant mass of the six-pion system; $d N_{4 \pi 2 \pi^{0} \gamma}$ is the number of selected six-pion events after background subtraction in the interval $d E_{\text {c.m. }}$ and $\epsilon_{4 \pi 2 \pi^{0}}^{\mathrm{MC}}\left(E_{\text {c.m. }}\right)$ is the corresponding detection efficiency obtained from the MC simulation. The factor $\epsilon_{4 \pi 2 \pi^{0}}^{\text {corr }}$ takes into account the differences between data and MC in tracking and $\pi^{0}$ efficiencies, as discussed in Sec. V C.

The energy dependence of the cross section for the reaction $e^{+} e^{-} \rightarrow 2\left(\pi^{+} \pi^{-} \pi^{0}\right)$ after all corrections is shown in Fig. 13. It again shows a structure around $1.9 \mathrm{GeV}$, reaching a peak value of about $6 \mathrm{nb}$ near 2.0 GeV. The cross section for each $25 \mathrm{MeV}$ interval is presented in Table III.

Since $d \mathcal{L}$ [see Eq. (2)] has been corrected for vacuum polarization and final-state photon emission, the cross section includes effects due to vacuum polarization and final-state soft-photon emission. For $g-2$ calculations, vacuum polarization contributions should be excluded from our data.

The observed line shape is not purely Gaussian mainly due to soft-photon radiation. Once again, no unfolding of the resolution is performed for the results shown here. The coefficients in Eq. (4) appropriate to the $2\left(\pi^{+} \pi^{-} \pi^{0}\right)$ case are $e 1, \ldots, e 5=0.007,0.091,0.744,0.114,0.011$.

\section{F. Summary of systematic studies}

The measured cross sections for the $2\left(\pi^{+} \pi^{-} \pi^{0}\right)$ final state, shown in Fig. 13 and tabulated in Table III, include only statistical errors. The systematic errors discussed in previous sections are summarized in Table IV, along with

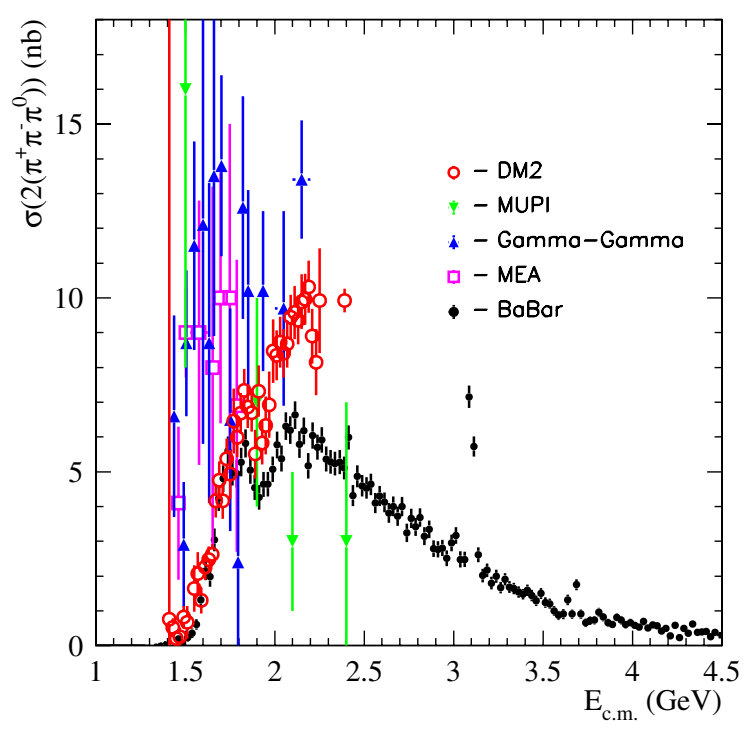

FIG. 13 (color online). The $e^{+} e^{-}$c.m. energy dependence of the $2\left(\pi^{+} \pi^{-} \pi^{0}\right)$ cross section measured with ISR data at BABAR compared with the direct $e^{+} e^{-}$measurements by detectors at ADONE and DCI. Only statistical errors are shown. 
TABLE III. Summary of the $e^{+} e^{-} \rightarrow 2\left(\pi^{+} \pi^{-} \pi^{0}\right)$ cross section measurement. Errors are statistical only.

\begin{tabular}{|c|c|c|c|c|c|c|c|}
\hline$E_{\text {c.m. }}(\mathrm{GeV})$ & $\sigma(\mathrm{nb})$ & $E_{\text {c.m. }}(\mathrm{GeV})$ & $\sigma(\mathrm{nb})$ & $E_{\text {c.m. }}(\mathrm{GeV})$ & $\sigma(\mathrm{nb})$ & $E_{\text {c.m. }}(\mathrm{GeV})$ & $\sigma(\mathrm{nb})$ \\
\hline 1.3125 & $0.00 \pm 0.04$ & 2.1125 & $6.44 \pm 0.43$ & 2.9125 & $2.46 \pm 0.26$ & 3.7125 & $0.74 \pm 0.15$ \\
\hline 1.3375 & $0.00 \pm 0.05$ & 2.1375 & $5.45 \pm 0.42$ & 2.9375 & $2.49 \pm 0.26$ & 3.7375 & $0.51 \pm 0.13$ \\
\hline 1.3625 & $0.04 \pm 0.06$ & 2.1625 & $5.92 \pm 0.41$ & 2.9625 & $2.16 \pm 0.26$ & 3.7625 & $0.60 \pm 0.14$ \\
\hline 1.3875 & $0.06 \pm 0.07$ & 2.1875 & $4.81 \pm 0.40$ & 2.9875 & $2.64 \pm 0.26$ & 3.7875 & $0.64 \pm 0.13$ \\
\hline 1.4125 & $0.09 \pm 0.08$ & 2.2125 & $5.81 \pm 0.40$ & 3.0125 & $2.88 \pm 0.26$ & 3.8125 & $0.86 \pm 0.14$ \\
\hline 1.4375 & $0.00 \pm 0.06$ & 2.2375 & $5.45 \pm 0.39$ & 3.0375 & $2.01 \pm 0.26$ & 3.8375 & $0.73 \pm 0.13$ \\
\hline 1.4625 & $0.19 \pm 0.09$ & 2.2625 & $5.68 \pm 0.39$ & 3.0625 & $2.17 \pm 0.24$ & 3.8625 & $0.53 \pm 0.13$ \\
\hline 1.4875 & $0.04 \pm 0.09$ & 2.2875 & $5.04 \pm 0.39$ & 3.0875 & $6.94 \pm 0.34$ & 3.8875 & $0.53 \pm 0.12$ \\
\hline 1.5125 & $0.18 \pm 0.13$ & 2.3125 & $5.10 \pm 0.36$ & 3.1125 & $5.48 \pm 0.31$ & 3.9125 & $0.70 \pm 0.13$ \\
\hline 1.5375 & $0.30 \pm 0.15$ & 2.3375 & $4.99 \pm 0.37$ & 3.1375 & $2.41 \pm 0.23$ & 3.9375 & $0.62 \pm 0.13$ \\
\hline 1.5625 & $0.58 \pm 0.16$ & 2.3625 & $5.01 \pm 0.37$ & 3.1625 & $1.77 \pm 0.22$ & 3.9625 & $0.50 \pm 0.12$ \\
\hline 1.5875 & $1.29 \pm 0.22$ & 2.3875 & $4.88 \pm 0.36$ & 3.1875 & $2.01 \pm 0.21$ & 3.9875 & $0.55 \pm 0.12$ \\
\hline 1.6125 & $2.10 \pm 0.28$ & 2.4125 & $5.86 \pm 0.37$ & 3.2125 & $1.63 \pm 0.20$ & 4.0125 & $0.47 \pm 0.12$ \\
\hline 1.6375 & $1.72 \pm 0.32$ & 2.4375 & $4.07 \pm 0.33$ & 3.2375 & $1.79 \pm 0.21$ & 4.0375 & $0.42 \pm 0.12$ \\
\hline 1.6625 & $2.88 \pm 0.35$ & 2.4625 & $4.70 \pm 0.34$ & 3.2625 & $1.52 \pm 0.18$ & 4.0625 & $0.60 \pm 0.12$ \\
\hline 1.6875 & $4.12 \pm 0.39$ & 2.4875 & $4.37 \pm 0.33$ & 3.2875 & $1.77 \pm 0.19$ & 4.0875 & $0.40 \pm 0.11$ \\
\hline 1.7125 & $4.67 \pm 0.41$ & 2.5125 & $4.25 \pm 0.34$ & 3.3125 & $1.54 \pm 0.19$ & 4.1125 & $0.50 \pm 0.11$ \\
\hline 1.7375 & $5.42 \pm 0.45$ & 2.5375 & $4.39 \pm 0.33$ & 3.3375 & $1.50 \pm 0.18$ & 4.1375 & $0.46 \pm 0.11$ \\
\hline 1.7625 & $4.86 \pm 0.41$ & 2.5625 & $3.85 \pm 0.31$ & 3.3625 & $1.40 \pm 0.18$ & 4.1625 & $0.32 \pm 0.11$ \\
\hline 1.7875 & $4.65 \pm 0.43$ & 2.5875 & $4.13 \pm 0.31$ & 3.3875 & $1.38 \pm 0.17$ & 4.1875 & $0.45 \pm 0.10$ \\
\hline 1.8125 & $4.97 \pm 0.44$ & 2.6125 & $3.92 \pm 0.31$ & 3.4125 & $1.46 \pm 0.17$ & 4.2125 & $0.17 \pm 0.10$ \\
\hline 1.8375 & $5.65 \pm 0.44$ & 2.6375 & $3.49 \pm 0.31$ & 3.4375 & $1.27 \pm 0.18$ & 4.2375 & $0.50 \pm 0.11$ \\
\hline 1.8625 & $4.86 \pm 0.41$ & 2.6625 & $3.74 \pm 0.31$ & 3.4625 & $1.14 \pm 0.17$ & 4.2625 & $0.14 \pm 0.10$ \\
\hline 1.8875 & $4.37 \pm 0.39$ & 2.6875 & $3.41 \pm 0.30$ & 3.4875 & $1.35 \pm 0.17$ & 4.2875 & $0.39 \pm 0.11$ \\
\hline 1.9125 & $4.05 \pm 0.38$ & 2.7125 & $3.72 \pm 0.30$ & 3.5125 & $1.06 \pm 0.17$ & 4.3125 & $0.26 \pm 0.10$ \\
\hline 1.9375 & $4.44 \pm 0.39$ & 2.7375 & $2.94 \pm 0.29$ & 3.5375 & $1.07 \pm 0.16$ & 4.3375 & $0.56 \pm 0.10$ \\
\hline 1.9625 & $4.48 \pm 0.38$ & 2.7625 & $3.34 \pm 0.30$ & 3.5625 & $0.87 \pm 0.15$ & 4.3625 & $0.26 \pm 0.11$ \\
\hline 1.9875 & $4.85 \pm 0.40$ & 2.7875 & $3.11 \pm 0.29$ & 3.5875 & $0.70 \pm 0.15$ & 4.3875 & $0.30 \pm 0.10$ \\
\hline 2.0125 & $5.59 \pm 0.41$ & 2.8125 & $3.43 \pm 0.29$ & 3.6125 & $0.70 \pm 0.16$ & 4.4125 & $0.34 \pm 0.10$ \\
\hline 2.0375 & $5.15 \pm 0.40$ & 2.8375 & $2.81 \pm 0.28$ & 3.6375 & $1.18 \pm 0.16$ & 4.4375 & $0.16 \pm 0.10$ \\
\hline 2.0625 & $6.12 \pm 0.42$ & 2.8625 & $3.12 \pm 0.27$ & 3.6625 & $0.74 \pm 0.15$ & 4.4625 & $0.32 \pm 0.10$ \\
\hline 2.0875 & $5.97 \pm 0.42$ & 2.8875 & $2.45 \pm 0.26$ & 3.6875 & $1.65 \pm 0.17$ & 4.4875 & $0.17 \pm 0.10$ \\
\hline
\end{tabular}

the corrections that were applied to the measurements. The two systematic corrections applied to the measured cross sections sum to $+8.8 \%$ with $10 \%-13 \%$ systematic uncertainty.

\section{G. Physics results}

The cross section for the $2\left(\pi^{+} \pi^{-} \pi^{0}\right)$ final state measured by $B A B A R$ (Fig. 13) can be compared with existing $e^{+} e^{-}$measurements performed by the $\mu \pi$ [21], Gamma-

TABLE IV. Summary of systematic errors for the $e^{+} e^{-} \rightarrow 2\left(\pi^{+} \pi^{-} \pi^{0}\right)$ cross section measurement.

\begin{tabular}{lcr}
\hline \hline Source & Correction applied & Systematic error \\
\hline Luminosity from $\mu \mu \gamma$ & $\cdots$ & $3 \%$ \\
MC-data difference in $\chi^{2}<40$ signal region & $0 \%$ & $6 \%$ \\
Background subtraction & $\cdots$ & $5 \%$ for $m_{2\left(\pi^{+} \pi^{-} \pi^{0}\right)<3.0 \mathrm{GeV} / \mathrm{c}^{2}}$ \\
& & $10 \%$ for $m_{2\left(\pi^{+} \pi^{-} \pi^{0}\right)}>3.0 \mathrm{GeV} / \mathrm{c}^{2}$ \\
MC-data difference in tracking efficiency & $2 \%$ & $3 \%$ \\
MC-data difference in $\pi^{0}$ losses & $+3 \%$ & $1 \%$ \\
Radiative corrections accuracy & $\cdots .6 \%$ & $3 \%$ \\
Acceptance from MC (model-dependent) & $\cdots$ & $10 \%$ for $m_{2\left(\pi^{+} \pi^{-} \pi^{0}\right)}<3.0 \mathrm{GeV} / \mathrm{c}^{2}$ \\
Total (assuming addition in quadrature and no correlations) & $+8.8 \%$ & $13 \%$ for $m_{2\left(\pi^{+} \pi^{-} \pi^{0}\right)}>3.0 \mathrm{GeV} / \mathrm{c}^{2}$ \\
\hline \hline
\end{tabular}


THE $e^{+} e^{-} \rightarrow 3\left(\pi^{+} \pi^{-}\right), 2\left(\pi^{+} \pi^{-} \pi^{0}\right)$ AND $K^{+} K^{-} 2\left(\pi^{+} \pi^{-}\right) \ldots$
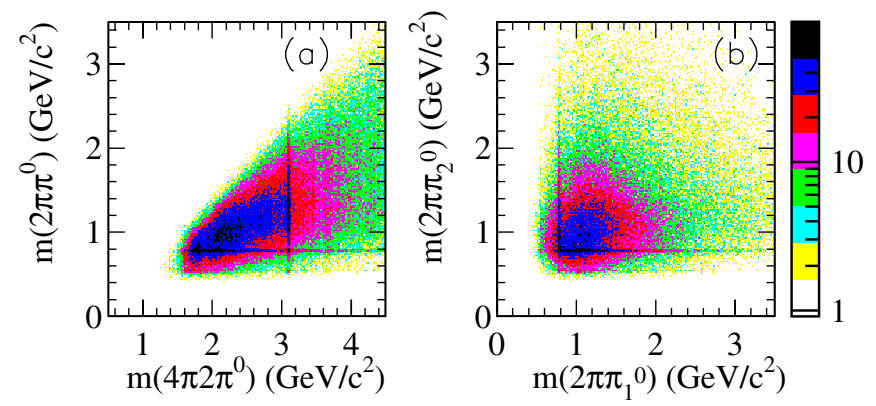

FIG. 14 (color online). Invariant-mass scatter plots from data for (a) the six-pion $\left[2\left(\pi^{+} \pi^{-} \pi^{0}\right)\right]$ final state versus the neutral three-pion combinations $\left(\pi^{+} \pi^{-} \pi^{0}\right)$, and (b) one neutral threepion combination with the lower momentum $\pi^{0}$ versus the other with the higher momentum $\pi^{0}$.

Gamma [22] and MEA [23] detectors at the ADONE collider and by the DM2 [18] detector at DCI. The $B A B A R$ measurement is much more precise and disagrees with DM2. The latter probably have large systematic errors due to normalization, not discussed in the original reference [18] that mostly focuses on the confirmation of the dip at $1.9 \mathrm{GeV}$ previously observed in the six-charged-pion mode.

Different mass combinations were studied in data and MC events to search for any structures or states not included in the simulation.

Figure 14 shows invariant-mass scatter plots for (a) neutral three- versus six-pion combinations, and (b) neutral $\pi^{+} \pi^{-} \pi^{0}$ combinations with the lower momentum $\pi^{0}$ versus three-pion with the higher momentum $\pi^{0}$. The $\omega(782)$ and $\eta$ mesons are seen in the three-body combinations. Figure 15(a) shows the projection of the three-pion invariant-mass distribution of Fig. 14 with clear $\omega(782)$ and $\eta$ signals. Figures 15(b)-15(d) show mass projections for the two-pion combinations. Backgrounds are subtracted from the data points shown using the $\chi^{2}$ control region, and the non-ISR JETSET simulation (shaded histograms in Fig. 15), as described above. The $\rho(770)$ meson is clearly seen in the $\pi^{ \pm} \pi^{0}$ and $\pi^{+} \pi^{-}$combinations, while there is some indication of a small signal from $f_{0}(980)$ in the $\pi^{0} \pi^{0}$ mass distribution. The phase space MC simulation shown by unshaded histograms in Fig. 15 does not include any of these structures.

Figure 16 compares the $\pi^{+} \pi^{-}$mass distribution (multiplied by two) with the summed $\pi^{ \pm} \pi^{0}$ mass distribution. The basic shapes are quite similar, although there are more than twice as many charged as neutral $\rho(770)$ mesons. The neutral $\pi^{+} \pi^{-}$also has a broad bump around $1.3 \mathrm{GeV} / \mathrm{c}^{2}$ relative to the charged $\pi^{ \pm} \pi^{0}$ distribution, perhaps indicating the presence of some intermediate $f_{0}(1370)$ or $f_{2}(1270)$ production.

No obvious structures are seen in the four- or five-pion combinations (not shown). Though the structures observed above are suggestive, a partial wave analysis would be needed to interpret the data more completely.
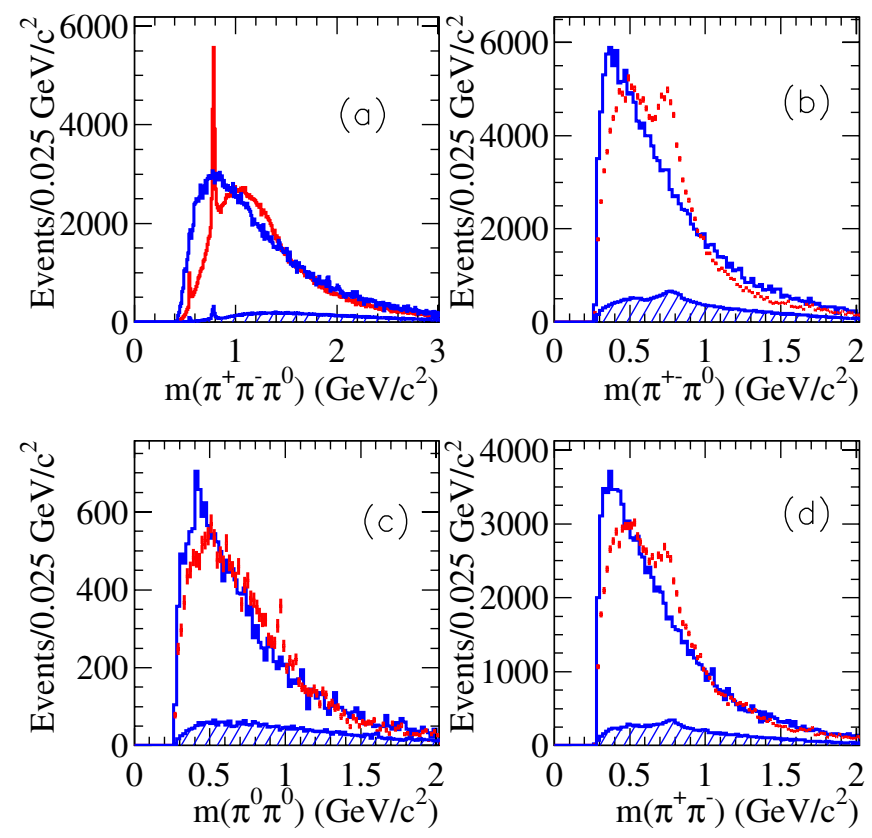

FIG. 15 (color online). Invariant-mass distributions from data (points), simulation (unshaded histograms), and the non-ISR contributions obtained from JETSET (shaded histograms) for (a) neutral three-pion $\left(\pi^{+} \pi^{-} \pi^{0}\right)$ combinations, (b) charged two-pion $\left(\pi^{ \pm} \pi^{0}\right)$ combinations, (c) neutral two-body pi-zero $\left(\pi^{0} \pi^{0}\right)$ combinations, and (d) neutral two-body charged-pion $\left(\pi^{+} \pi^{-}\right)$combinations.

Estimates for the $\omega(782)$ and $\eta$ contributions can be obtained using the $\pi^{+} \pi^{-} \pi^{0}$ mass distribution of Fig. 17(a) which shows an expanded view of Fig. 15(a). The procedure is to fit each signal with a two-Gaussian function representing the signal plus a polynomial background,

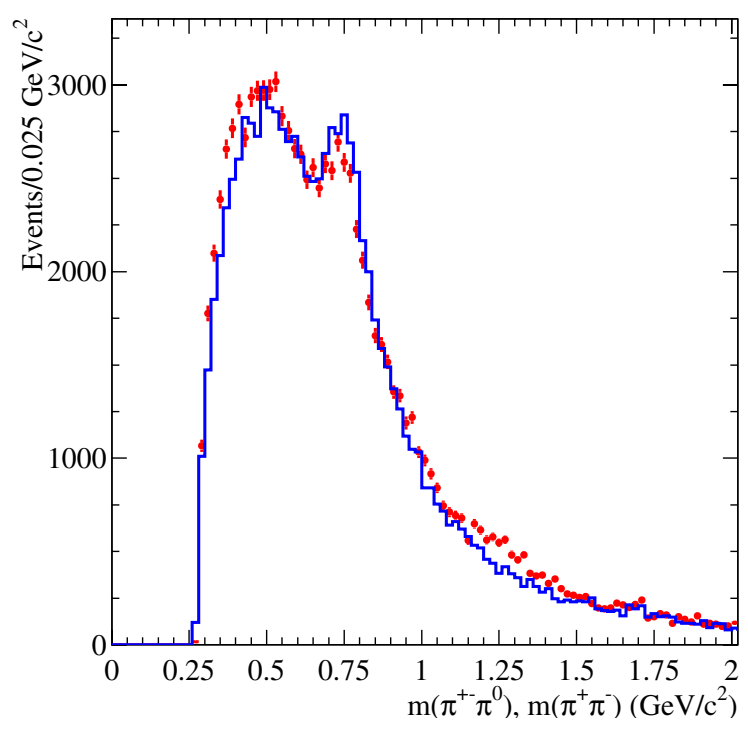

FIG. 16 (color online). The scaled $\pi^{+} \pi^{-}$mass distribution (points, with errors, multiplied by two) compared with the summed $\pi^{ \pm} \pi^{0}$ mass distribution (histogram). 

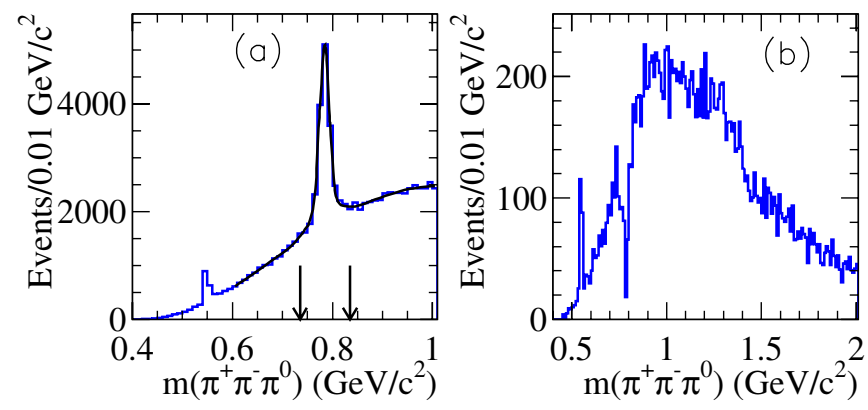

FIG. 17 (color online). The $\pi^{+} \pi^{-} \pi^{0}$ invariant-mass distributions (a) in the low-mass region, and (b) for the second $\pi^{+} \pi^{-} \pi^{0}$ invariant-mass combination remaining in those events where the first combination lies within the mass range indicated by the arrows in (a).

yielding $9899 \pm 158$ and $770 \pm 40, \omega \pi^{+} \pi^{-} \pi^{0}$ and $\eta \pi^{+} \pi^{-} \pi^{0}$ events, respectively.

The energy dependence of the $\omega \pi^{+} \pi^{-} \pi^{0}$ cross section is determined by performing this fit for each $25 \mathrm{MeV} / \mathrm{c}^{2}$ bin of the six-pion $\left[2\left(\pi^{+} \pi^{-} \pi^{0}\right)\right]$ mass (Fig. 18). In addition to the broad structure at low mass, there is also a sharp structure in the $J / \psi$ region corresponding to $170 \pm 24$ events decaying into the $\omega \pi^{+} \pi^{-} \pi^{0}$ final state. After correcting for efficiency and normalizing to the ISR luminosity, this yields the $e^{+} e^{-} \rightarrow \omega \pi^{+} \pi^{-} \pi^{0}$ cross section shown by the squares in Fig. 18.

Six-pion events that contain an $\omega$, defined by the arrows in Fig. 17(a), also sometimes contain an $\eta$ as shown in Fig. 17(b). After selecting these $\omega \eta$ events, and subtracting the background using the $\eta$ side bands, we calculate the cross section for $e^{+} e^{-} \rightarrow \omega \eta$ presented in Fig. 19. The cross section is corrected for the decay rate of $\omega$ and $\eta$ to

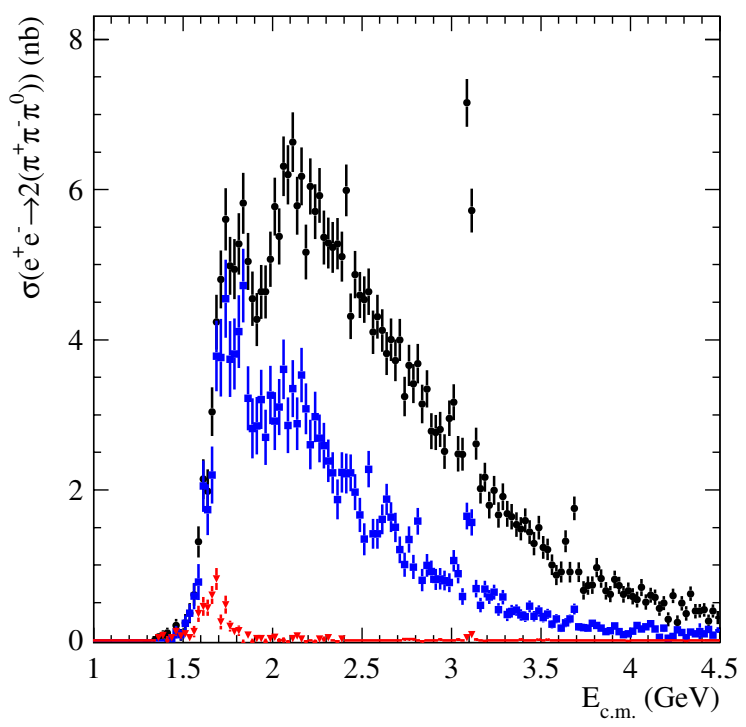

FIG. 18 (color online). The cross section versus $E_{\text {c.m. }}$ for all $2\left(\pi^{+} \pi^{-} \pi^{0}\right)$ events (circles), $\omega \pi^{+} \pi^{-} \pi^{0}$ events (squares), and $\omega \eta$ events (triangles).

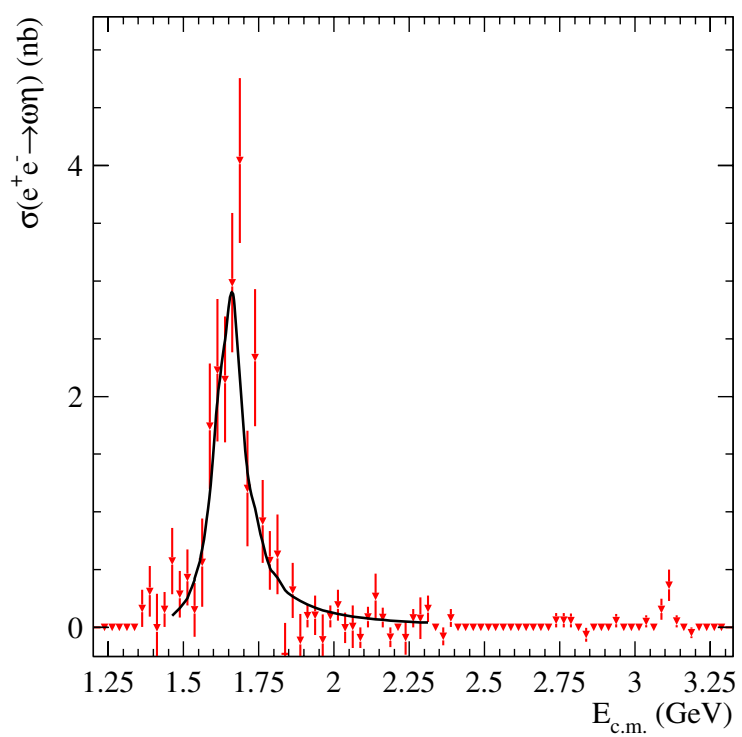

FIG. 19 (color online). The $\omega \eta$ cross section from the $2\left(\pi^{+} \pi^{-} \pi^{0}\right)$ event sample. The line is the fit to the structure in the $1.6 \mathrm{GeV}$ region described in the text.

$\pi^{+} \pi^{-} \pi^{0}$ taken from PDG [24]. A prominent structure can be seen around $1.6 \mathrm{GeV}$, with a smaller peak (from 13 events over a background of less than 0.5 ) around $3.1 \mathrm{GeV}$ from $J / \psi \rightarrow \omega \eta$ decay. The observed cross section is fitted with a resonance-type parametrization

$$
\begin{gathered}
\sigma\left(e^{+} e^{-} \rightarrow \omega \eta\right)=\frac{F(s)}{s^{3 / 2}}\left|A_{m}(s)\right|^{2}, \\
A_{m}(s)=\frac{m^{5 / 2} \Gamma_{0} \sqrt{\sigma_{0} / F\left(m^{2}\right)}}{s-m^{2}+i \sqrt{s} \Gamma_{0}},
\end{gathered}
$$

where $m$ is the mass, $\Gamma_{0}$ the width, and $\sigma_{0}$ the peak cross section of this resonance production in $e^{+} e^{-}$collisions. $F(s)$ is a phase space term equal to the cube of the $\omega$ (or $\eta$ ) momentum in the $\omega \eta$ rest frame. The fit gives $m=$ $1.645 \pm 0.008 \mathrm{GeV} / \mathrm{c}^{2}, \quad \Gamma_{0}=0.114 \pm 0.014 \mathrm{GeV}$, and $\sigma_{0}=3.08 \pm 0.33 \mathrm{nb}$.

As discussed in Sec. VE, the effect of mass resolution on the measured width of this structure is small and not taken into account. The mass value obtained is close to the value $1670 \pm 30 \mathrm{GeV} / \mathrm{c}^{2}$ listed in the PDG [24] for the $\omega(1650)$, for which the $\omega \eta$ final state has been seen by earlier experiments, but the width seen here is substantially narrower than the $0.315 \pm 0.035 \mathrm{GeV}$ listed. A structure decaying to $\omega \eta$ in this region might also correspond to the $\phi(1680)$, whose mass and width are listed in the PDG as $1.680 \pm 0.020 \mathrm{GeV} / \mathrm{c}^{2}$ and $0.150 \pm 0.050 \mathrm{GeV}$, respectively. However, no branching fraction of $\phi(1680)$ to the $\omega \eta$ final state was reported in the previous experiments.

Figure 20(a) shows the cross section ratio for $3\left(\pi^{+} \pi^{-}\right)$ to $2\left(\pi^{+} \pi^{-} \pi^{0}\right)$, as given by Figs. 6 and 18, respectively. A 
THE $e^{+} e^{-} \rightarrow 3\left(\pi^{+} \pi^{-}\right), 2\left(\pi^{+} \pi^{-} \pi^{0}\right)$ AND $K^{+} K^{-} 2\left(\pi^{+} \pi^{-}\right) \ldots$
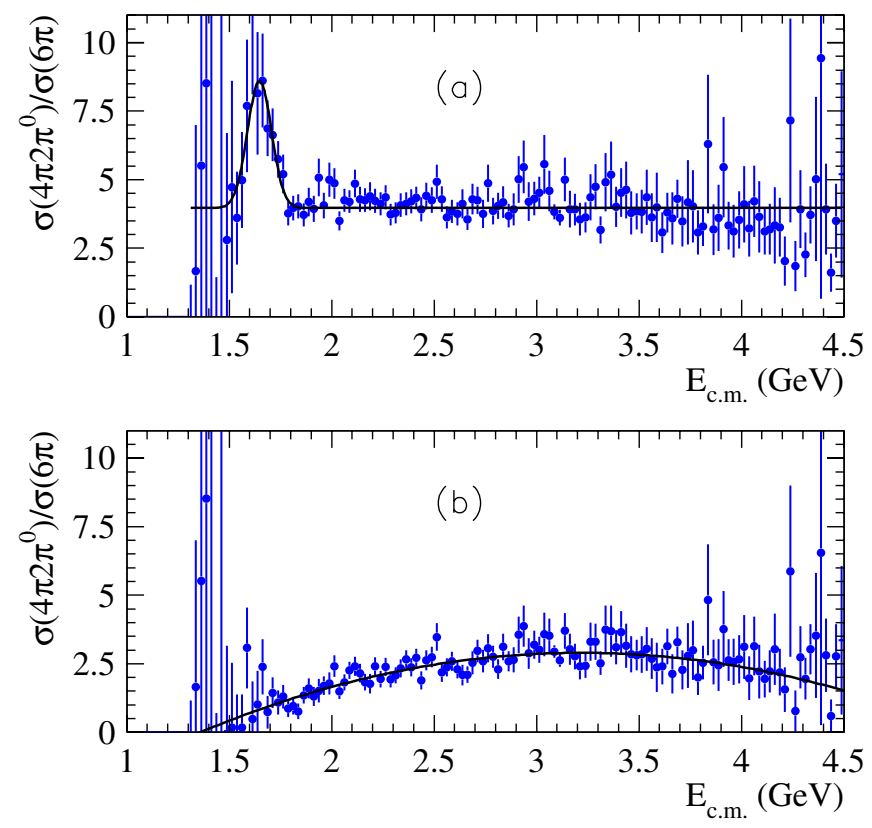

FIG. 20 (color online). Ratio of the $2\left(\pi^{+} \pi^{-} \pi^{0}\right)$ to $3\left(\pi^{+} \pi^{-}\right)$ cross sections for (a) selected events, and (b) the same events excluding the $\omega \pi^{+} \pi^{-} \pi^{0}$ contribution from the $2\left(\pi^{+} \pi^{-} \pi^{0}\right)$ sample. The lines are fits described in the text.

good fit to this ratio for all energy intervals, shown by the curve in Fig. 20(a), is obtained with a constant plus a Gaussian in the $1.6 \mathrm{GeV}$ region. The ratio equals $3.98 \pm$ $0.06 \pm 0.41$ everywhere, except for the region around $1.6 \mathrm{GeV}$, where it reaches about 8 at peak. The structure may be at least partially explained by the presence of the $\omega \eta$ structure in the $2\left(\pi^{+} \pi^{-} \pi^{0}\right)$ final state. When the $\omega \pi^{+} \pi^{-} \pi^{0}$ contribution is subtracted from the $2\left(\pi^{+} \pi^{-} \pi^{0}\right)$ final state, the ratio is no longer flat, as shown by Fig. 20(b), and the structure at low mass disappears. A 2nd-order polynomial fits the data well as shown by the curve in Fig. 20(b).

Figures 21 and 22 compare the $3\left(\pi^{+} \pi^{-}\right)$and $2\left(\pi^{+} \pi^{-} \pi^{0}\right)$ cross section data, respectively, with fits to the model presented in Ref. [20]. The structures observed in both channels around $1.9 \mathrm{GeV}$ are not well described by a single Breit-Wigner resonance, and may result from rather complicated physics, such as several vector states decaying to the same mode. The model [25] has the form

$$
\sigma_{6 \pi}=\frac{4 \pi \alpha^{2}}{s^{3 / 2}}\left(\frac{g m^{2} e^{i \phi}}{s-m^{2}+i \sqrt{s} \Gamma}+A_{\text {cont }}\right)^{2},
$$

where $m, \Gamma$ and $\phi$ are the mass, width and relative phase of the Breit-Wigner type amplitude, representing the structure; $g$ is a coupling constant and $A_{\text {cont }}=c_{0}+$ $c_{1}\left[e^{-b /\left(\sqrt{s}-m_{0}\right)} /\left(\sqrt{s}-m_{0}\right)^{2-a}\right]$ is a Jacob-Slansky amplitude [26] representing an amalgamation of broad resonances with $c_{0}, c_{1}, a, b, m_{0}$ free parameters. The following "resonance" parameters are obtained for the
PHYSICAL REVIEW D 73, 052003 (2006)

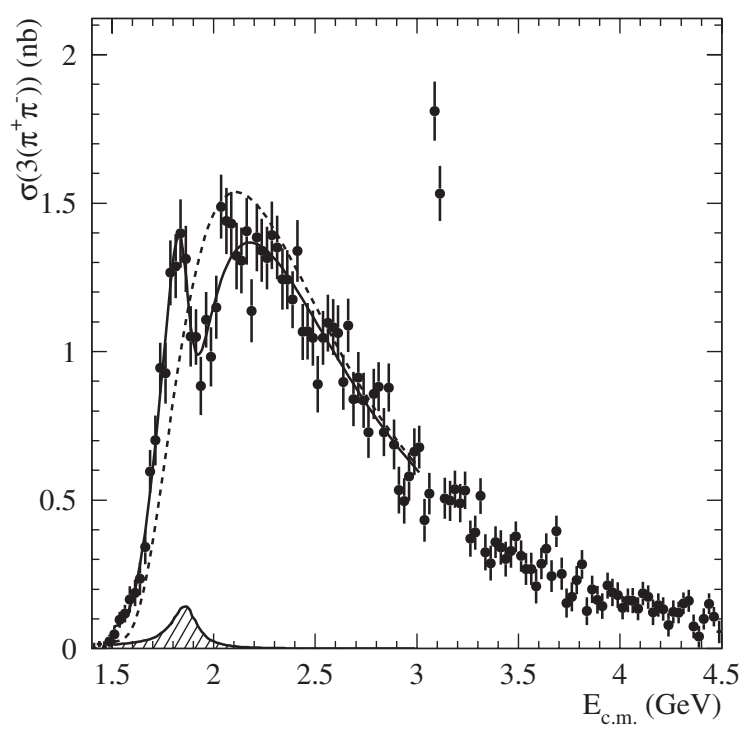

FIG. 21. The $3\left(\pi^{+} \pi^{-}\right)$cross section data, as given in Fig. 6, compared with the coherent fit (solid line) between resonance and continuum terms described in the text. The dashed line and the shaded function show the individual incoherent contributions from continuum and resonance terms.

structure:

$$
\begin{aligned}
& m_{6 \pi}=1.88 \pm 0.03 \mathrm{GeV} / \mathrm{c}^{2} \\
& m_{4 \pi 2 \pi^{0}}=1.86 \pm 0.02 \mathrm{GeV} / \mathrm{c}^{2}
\end{aligned}
$$

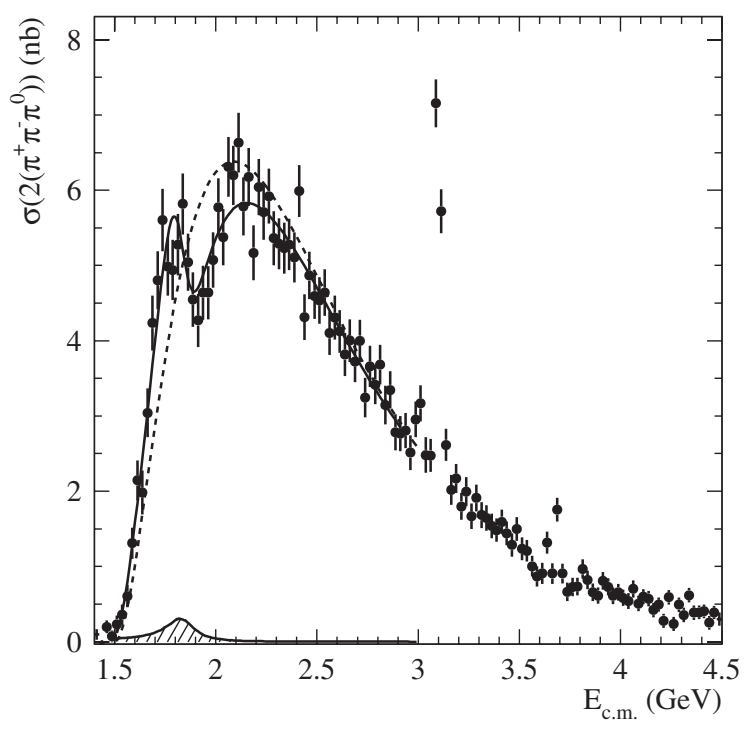

FIG. 22. The $2\left(\pi^{+} \pi^{-} \pi^{0}\right)$ cross section data, as given in Fig. 18, compared with the coherent fit (solid line) between resonance and continuum terms described in the text. The dashed line and the shaded function show the individual incoherent contributions from continuum and resonance terms. 


$$
\begin{array}{cc}
\Gamma_{6 \pi}=0.13 \pm 0.03 \mathrm{GeV} ; & \Gamma_{4 \pi 2 \pi^{0}}=0.16 \pm 0.02 \mathrm{GeV} \\
\phi_{6 \pi}=21^{\circ} \pm 40^{\circ} ; & \phi_{4 \pi 2 \pi^{0}}=-3^{\circ} \pm 15^{\circ} .
\end{array}
$$

The parameter values obtained seem to be essentially independent of the final-state charge combination. These values may also be compared with those obtained with a similar model by the FOCUS experiment [20,27] $m=$ $1.91 \pm 0.01 \mathrm{GeV} / \mathrm{c}^{2}, \quad \Gamma=0.037 \pm 0.013 \mathrm{GeV}, \quad \phi=$ $10^{\circ} \pm 30^{\circ}$. The mass values are consistent, but the widths obtained by $B A B A R$ are substantially larger.

\section{THE $K^{+} K^{-} 2\left(\pi^{+} \pi^{-}\right)$FINAL STATE}

The constrained fit of the six-charged-track events to the hypothesis of two oppositely charged kaons and fourcharged pions, where at least one of the kaons has positive particle identification, allows us to select this final state. Figure 23 shows the $\chi^{2}$ distributions for both data and simulation, where the simulation of the $K^{+} K^{-} 2\left(\pi^{+} \pi^{-}\right)$ reaction uses a phase space model with a cross section energy dependence close to that which we observe experimentally, and ISR and FSR extra radiative processes are included. Also shown is the estimated contribution from non-ISR events obtained by JETSET simulation.

Figure 24(a) presents the simulated mass distribution for the $2 K 4 \pi$ events. The mass dependence of the efficiency, calculated as a ratio of selected to generated $2 K 4 \pi \mathrm{MC}$ events, is shown in Fig. 24(b). The efficiency falls gradu-

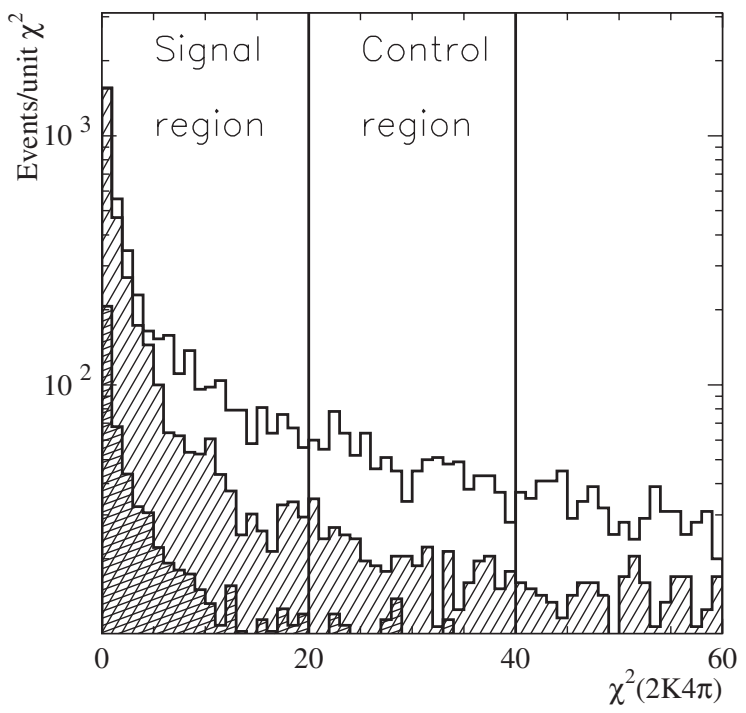

FIG. 23. The one-constraint $\chi^{2}$ distributions for data (unshaded histogram) and $\mathrm{MC} K^{+} K^{-} 2\left(\pi^{+} \pi^{-}\right) \gamma$ simulation (shaded histogram) for six-charged-track events fitted to the $K^{+} K^{-} 2\left(\pi^{+} \pi^{-}\right)$hypothesis. The cross-hatched histogram is the estimated background contribution from non-ISR events obtained from JETSET. The signal and control regions are indicated.
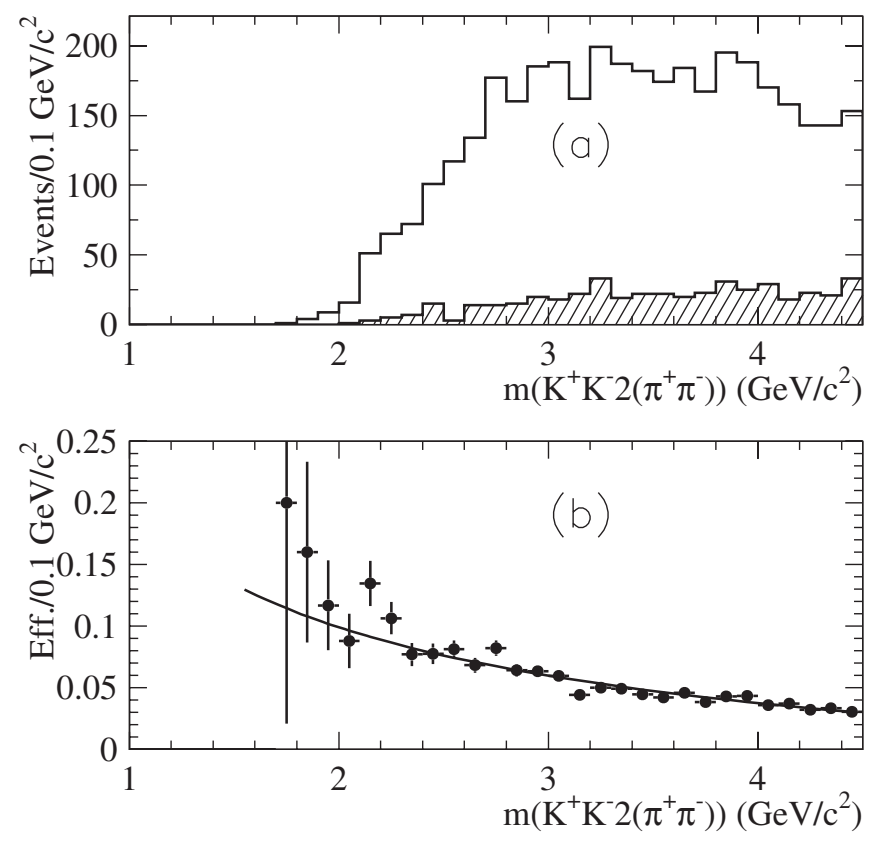

FIG. 24. (a) The $K^{+} K^{-} 2\left(\pi^{+} \pi^{-}\right)$mass distributions from MC simulation for the signal (unshaded) and control (shaded) regions of Fig. 23. (b) The mass dependence of the net reconstruction and selection efficiency obtained from simulation. The curve is a fit described in the text.

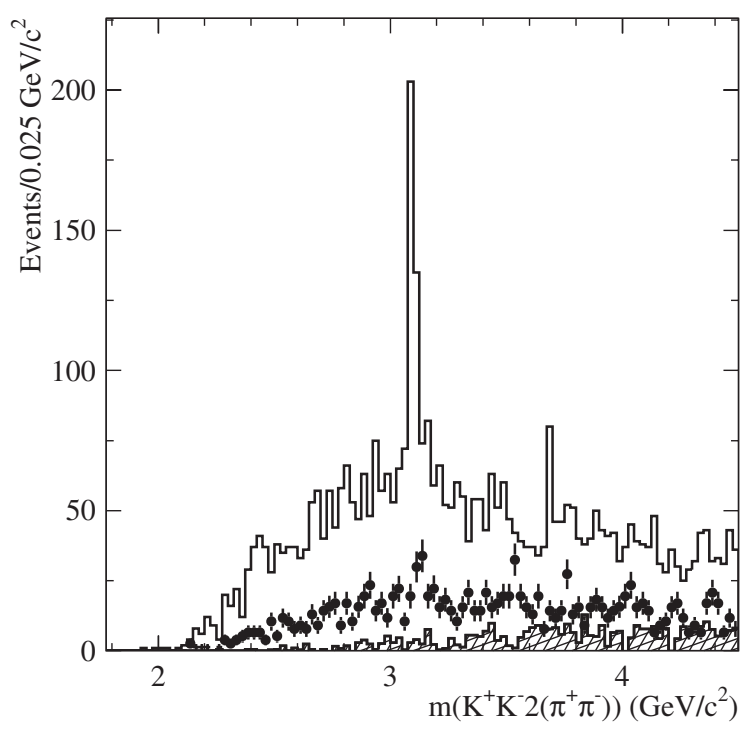

FIG. 25. The $K^{+} K^{-} 2\left(\pi^{+} \pi^{-}\right)$invariant-mass distribution (unshaded histogram) for the signal region of Fig. 23, after subtracting misidentified six-charged-pion events as described in the text. The points indicate the background estimated from the difference between data and MC events for the control region of Fig. 23, normalized to the difference between data and MC events in the signal region of Fig. 23. The crosshatched histogram corresponds to the non-ISR background of Fig. 23. 


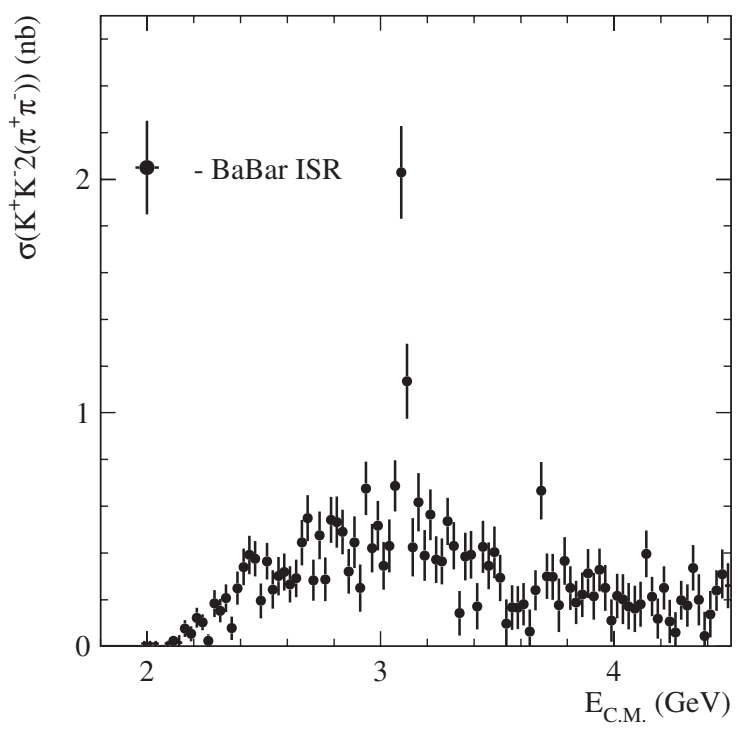

FIG. 26. The c.m. energy dependence of the $e^{+} e^{-} \rightarrow$ $K^{+} K^{-} 2\left(\pi^{+} \pi^{-}\right)$cross section obtained from ISR events at $B A B A R$. Only statistical errors are shown.

ally from about $15 \%$ at low mass, to about $3 \%$ at $4.5 \mathrm{GeV} / \mathrm{c}^{2}$. The data are well represented by the 3rd-order polynomial shown by the curve in Fig. 24(b). After the selections $\chi_{2 K 4 \pi}^{2}<20, \chi_{6 \pi}^{2}>20$ are imposed, we estimate from simulation that about $0.6 \%$ of the six-charged-pion events remain in the sample due to misidentification of pions. Although the background subtraction procedure, as described above, using the control region $20<\chi_{2 K 4 \pi}^{2}<40$ is invoked, background from pion misidentification will not be subtracted by this procedure. Thus, $0.6 \%$ of the sixpion events shown in Fig. 2 are subtracted, leading to a correction of about $3 \%$ to the total number of $K^{+} K^{-} 2\left(\pi^{+} \pi^{-}\right)$events.

Figure 25 shows the $K^{+} K^{-} 2\left(\pi^{+} \pi^{-}\right)$invariant-mass distributions for signal events, selected as defined above, as well as for events from the control region in $\chi_{2 K 4 \pi}^{2}$, and for the expected non-ISR background from JETSET MC simulation. These latter two distributions are used to subtract background in the signal region. Clear $J / \psi$ and $\psi(2 S)$ signals are seen.

Using the number of observed events, efficiency, and ISR luminosity, we obtain the $e^{+} e^{-} \rightarrow K^{+} K^{-} 2\left(\pi^{+} \pi^{-}\right)$ cross section shown in Fig. 26. No measurements are available from earlier experiments. Table $\mathrm{V}$ presents the cross section in $25 \mathrm{MeV}$ bins. The systematic errors are dominated by the uncertainty in the acceptance simulation $(10 \%)$, by the uncertainty in the background subtraction (5\%), and by the difference between the kaon identification efficiencies for data and MC events (up to $2 \%$ per track), and are estimated to be about $15 \%$.

TABLE V. Summary of the $e^{+} e^{-} \rightarrow K^{+} K^{-} 2\left(\pi^{+} \pi^{-}\right)$cross section measurement. Errors are statistical only.

\begin{tabular}{|c|c|c|c|c|c|c|c|}
\hline$E_{\text {c.m. }}(\mathrm{GeV})$ & $\sigma(\mathrm{nb})$ & $E_{\text {c.m. }}(\mathrm{GeV})$ & $\sigma(\mathrm{nb})$ & $E_{\text {c.m. }}(\mathrm{GeV})$ & $\sigma(\mathrm{nb})$ & $E_{\text {c.m. }}(\mathrm{GeV})$ & $\sigma(\mathrm{nb})$ \\
\hline 2.0125 & $0.01 \pm 0.01$ & 2.6375 & $0.29 \pm 0.08$ & 3.2625 & $0.36 \pm 0.10$ & 3.8875 & $0.31 \pm 0.11$ \\
\hline 2.0375 & $0.01 \pm 0.01$ & 2.6625 & $0.44 \pm 0.10$ & 3.2875 & $0.54 \pm 0.10$ & 3.9125 & $0.21 \pm 0.10$ \\
\hline 2.0625 & $0.00 \pm 0.00$ & 2.6875 & $0.55 \pm 0.10$ & 3.3125 & $0.43 \pm 0.10$ & 3.9375 & $0.33 \pm 0.09$ \\
\hline 2.0875 & $0.01 \pm 0.01$ & 2.7125 & $0.28 \pm 0.09$ & 3.3375 & $0.14 \pm 0.10$ & 3.9625 & $0.25 \pm 0.10$ \\
\hline 2.1125 & $0.02 \pm 0.02$ & 2.7375 & $0.47 \pm 0.10$ & 3.3625 & $0.38 \pm 0.10$ & 3.9875 & $0.11 \pm 0.09$ \\
\hline 2.1375 & $0.02 \pm 0.03$ & 2.7625 & $0.29 \pm 0.09$ & 3.3875 & $0.39 \pm 0.10$ & 4.0125 & $0.22 \pm 0.09$ \\
\hline 2.1625 & $0.08 \pm 0.04$ & 2.7875 & $0.54 \pm 0.10$ & 3.4125 & $0.17 \pm 0.10$ & 4.0375 & $0.20 \pm 0.11$ \\
\hline 2.1875 & $0.05 \pm 0.03$ & 2.8125 & $0.53 \pm 0.11$ & 3.4375 & $0.43 \pm 0.11$ & 4.0625 & $0.17 \pm 0.10$ \\
\hline 2.2125 & $0.12 \pm 0.04$ & 2.8375 & $0.49 \pm 0.10$ & 3.4625 & $0.34 \pm 0.10$ & 4.0875 & $0.16 \pm 0.10$ \\
\hline 2.2375 & $0.10 \pm 0.03$ & 2.8625 & $0.32 \pm 0.10$ & 3.4875 & $0.40 \pm 0.11$ & 4.1125 & $0.18 \pm 0.10$ \\
\hline 2.2625 & $0.02 \pm 0.03$ & 2.8875 & $0.45 \pm 0.11$ & 3.5125 & $0.29 \pm 0.10$ & 4.1375 & $0.40 \pm 0.10$ \\
\hline 2.2875 & $0.18 \pm 0.06$ & 2.9125 & $0.25 \pm 0.10$ & 3.5375 & $0.10 \pm 0.11$ & 4.1625 & $0.21 \pm 0.08$ \\
\hline 2.3125 & $0.15 \pm 0.05$ & 2.9375 & $0.68 \pm 0.11$ & 3.5625 & $0.17 \pm 0.10$ & 4.1875 & $0.12 \pm 0.09$ \\
\hline 2.3375 & $0.21 \pm 0.06$ & 2.9625 & $0.42 \pm 0.10$ & 3.5875 & $0.17 \pm 0.09$ & 4.2125 & $0.25 \pm 0.09$ \\
\hline 2.3625 & $0.08 \pm 0.05$ & 2.9875 & $0.52 \pm 0.11$ & 3.6125 & $0.18 \pm 0.09$ & 4.2375 & $0.11 \pm 0.09$ \\
\hline 2.3875 & $0.25 \pm 0.07$ & 3.0125 & $0.34 \pm 0.10$ & 3.6375 & $0.06 \pm 0.09$ & 4.2625 & $0.06 \pm 0.09$ \\
\hline 2.4125 & $0.34 \pm 0.08$ & 3.0375 & $0.43 \pm 0.11$ & 3.6625 & $0.24 \pm 0.09$ & 4.2875 & $0.20 \pm 0.08$ \\
\hline 2.4375 & $0.39 \pm 0.08$ & 3.0625 & $0.69 \pm 0.11$ & 3.6875 & $0.67 \pm 0.12$ & 4.3125 & $0.17 \pm 0.09$ \\
\hline 2.4625 & $0.38 \pm 0.08$ & 3.0875 & $2.03 \pm 0.20$ & 3.7125 & $0.30 \pm 0.10$ & 4.3375 & $0.34 \pm 0.10$ \\
\hline 2.4875 & $0.19 \pm 0.07$ & 3.1125 & $1.14 \pm 0.16$ & 3.7375 & $0.30 \pm 0.10$ & 4.3625 & $0.20 \pm 0.11$ \\
\hline 2.5125 & $0.36 \pm 0.08$ & 3.1375 & $0.42 \pm 0.13$ & 3.7625 & $0.18 \pm 0.11$ & 4.3875 & $0.04 \pm 0.10$ \\
\hline 2.5375 & $0.24 \pm 0.08$ & 3.1625 & $0.62 \pm 0.12$ & 3.7875 & $0.37 \pm 0.10$ & 4.4125 & $0.14 \pm 0.10$ \\
\hline 2.5625 & $0.30 \pm 0.08$ & 3.1875 & $0.39 \pm 0.11$ & 3.8125 & $0.25 \pm 0.09$ & 4.4375 & $0.24 \pm 0.09$ \\
\hline 2.5875 & $0.32 \pm 0.08$ & 3.2125 & $0.56 \pm 0.11$ & 3.8375 & $0.19 \pm 0.09$ & 4.4625 & $0.31 \pm 0.11$ \\
\hline 2.6125 & $0.26 \pm 0.08$ & 3.2375 & $0.37 \pm 0.10$ & 3.8625 & $0.22 \pm 0.10$ & 4.4875 & $0.26 \pm 0.10$ \\
\hline
\end{tabular}



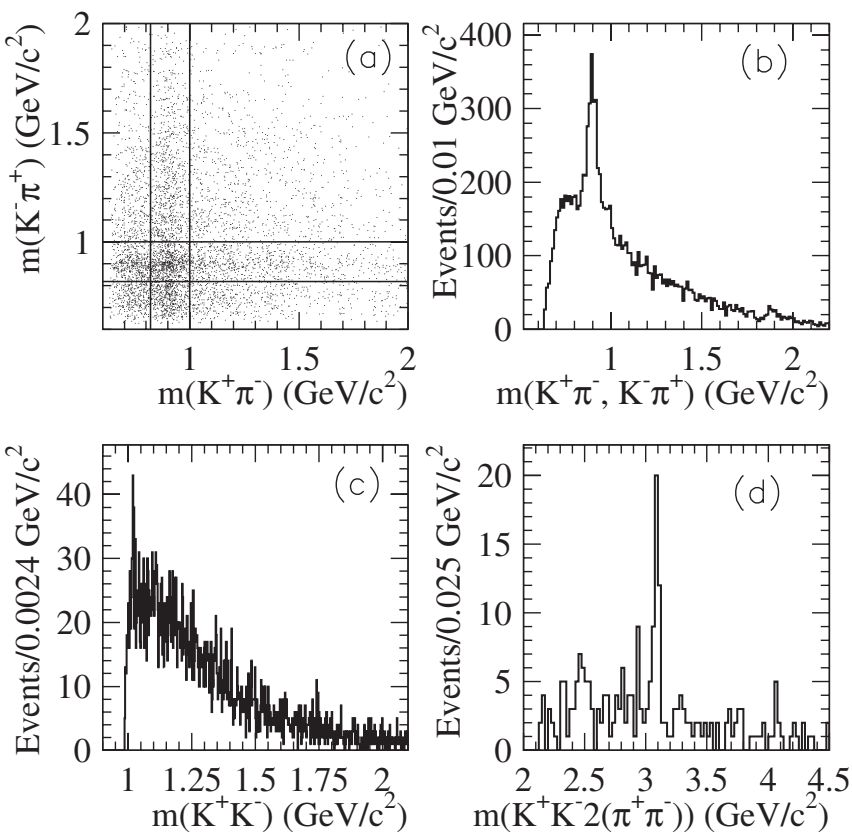

FIG. 27. Invariant-mass plots for various selections of data from the $K^{+} K^{-} 2\left(\pi^{+} \pi^{-}\right)$sample for (a) the scatter-plot of the $K^{+} \pi^{-}$and $K^{-} \pi^{+}$invariant-mass values; (b) the $K^{+} \pi^{-}$or $K^{-} \pi^{+}$mass projection of (a); (c) the mass distribution for $K^{+} K^{-}$; (d) the $K^{+} K^{-} 2\left(\pi^{+} \pi^{-}\right)$mass distribution for events around the $\phi(1020)$ peak from (c).

Figures 27(a) and 27(b) show the $K \pi$ mass combinations. Production of $K \pi$ pairs is dominated by the $K^{* 0}(892)$ clearly seen in Fig. 27(a) and the projection plot in Fig. 27(b). Some small structure is also seen at about $1.9 \mathrm{GeV} / \mathrm{c}^{2}$. Figure 27(c) shows the $K^{+} K^{-}$mass distribution. No structures except a small signal from $\phi(1020)$ are seen. These events correspond to $J / \psi \rightarrow$ $\phi(1020) 2\left(\pi^{+} \pi^{-}\right)$decay. This is demonstrated in Fig. $27(\mathrm{~d})$ where events from the $1.01-1.03 \mathrm{GeV} / \mathrm{c}^{2}$ mass interval of Fig. 27(c) are shown. We find $35 \pm 7$ events in the $J / \psi$ peak from the above decay chain.

\section{THE $J / \psi$ REGION}

Figure 28 shows an expanded view of the $J / \psi$ mass region in Fig. 2 for the six-pion data sample with no background subtraction. The signals from $J / \psi \rightarrow$ $3\left(\pi^{+} \pi^{-}\right)$and $\psi(2 S) \rightarrow 3\left(\pi^{+} \pi^{-}\right)$are clearly seen. The observation of $J / \psi$ decaying into six-charged pions gives a direct measurement of the $6 \pi$-mass resolution and the absolute energy scale. A fit with a Gaussian for the $J / \psi$ peak and a polynomial function for the continuum gives $\sigma_{m(6 \pi)}=8.7 \mathrm{MeV} / \mathrm{c}^{2}$ and less than $1 \mathrm{MeV} / \mathrm{c}^{2}$ difference from the PDG [24] value for the $J / \psi$ mass. The observed mass resolution agrees with the simulation within $10 \%$.

The observed $496 \pm 33$ events at the $J / \psi$ peak can be used to calculate the branching fraction for $J / \psi \rightarrow$ $3\left(\pi^{+} \pi^{-}\right)$. The simulation shows that because of radiative

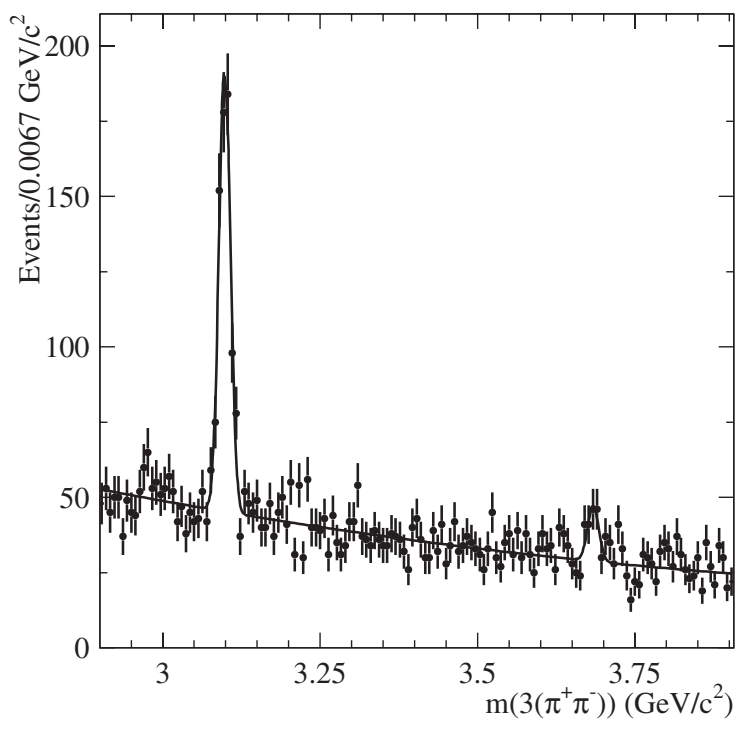

FIG. 28. The $3\left(\pi^{+} \pi^{-}\right)$mass distribution for ISR-produced $e^{+} e^{-} \rightarrow 3\left(\pi^{+} \pi^{-}\right)$events in the mass region around the $J / \psi$ and $\psi(2 S)$; there are clear signals at the $J / \psi$ and $\psi(2 S)$ mass positions.

effects only $90 \%$ of the signal events are under the Gaussian curve. Using the corrected number, we can calculate the products:

$$
\begin{aligned}
B_{J / \psi \rightarrow 6 \pi} \cdot \sigma_{\text {int }}^{J / \psi} & =\frac{N\left(J / \psi \rightarrow 3\left(\pi^{+} \pi^{-}\right)\right)}{d \mathcal{L} / d E \cdot \epsilon_{\mathrm{MC}}} \\
& =57.1 \pm 3.8 \pm 3.4 \mathrm{nb} \mathrm{MeV}, \\
B_{J / \psi \rightarrow 6 \pi} \cdot \Gamma_{e e}^{J / \psi} & =\frac{N\left(J / \psi \rightarrow 3\left(\pi^{+} \pi^{-}\right)\right) \cdot m_{J / \psi}^{2}}{6 \pi^{2} \cdot d \mathcal{L} / d E \cdot \epsilon_{\mathrm{MC}} \cdot C} \\
& =(2.37 \pm 0.16 \pm 0.14) \times 10^{-2} \mathrm{keV},
\end{aligned}
$$

where

$$
\sigma_{\text {int }}^{J / \psi}=6 \pi^{2} \Gamma_{e e}^{J / \psi} C / m_{J / \psi}^{2}=12983 \pm 409 \mathrm{nb} \mathrm{MeV}
$$

is the integral over the $J / \psi$ excitation curve, $\Gamma_{e e}^{J / \psi}$ is the electronic width, $d \mathcal{L} / d E=65.6 \mathrm{nb}^{-1} / \mathrm{MeV}$ is the ISR luminosity at the $J / \psi$ mass, $\epsilon_{\mathrm{MC}}=0.147$ is the detection efficiency from simulation with the corrections and errors discussed in Sec. IVF, and $C=3.894 \times 10^{11} \mathrm{nb} \mathrm{MeV}^{2}$ is a conversion constant. The subscript " $6 \pi$ " for branching fractions refers to the $3\left(\pi^{+} \pi^{-}\right)$final state exclusively.

Using $\Gamma_{e e}^{J / \psi}=5.40 \pm 0.18 \mathrm{keV}$ [24], we obtain $B_{J / \psi \rightarrow 6 \pi}=(4.40 \pm 0.29 \pm 0.29) \times 10^{-3}, \quad$ substantially more precise than the current PDG value $B_{J / \psi \rightarrow 6 \pi}=$ $(4.0 \pm 2.0) \times 10^{-3}[24]$. The systematic error includes a $3 \%$ uncertainty in $\Gamma_{e e}^{J / \psi}$.

The $\psi(2 S)$ peak mostly corresponds to the decay chain $\psi(2 S) \rightarrow J / \psi \pi^{+} \pi^{-} \rightarrow 3\left(\pi^{+} \pi^{-}\right)$. The number of events extracted from a fit to a Gaussian distribution for the $\psi(2 S)$ peak and a polynomial function for the continuum is $61 \pm$ 16 . The direct decay of $\psi(2 S) \rightarrow 3\left(\pi^{+} \pi^{-}\right)$is very small 


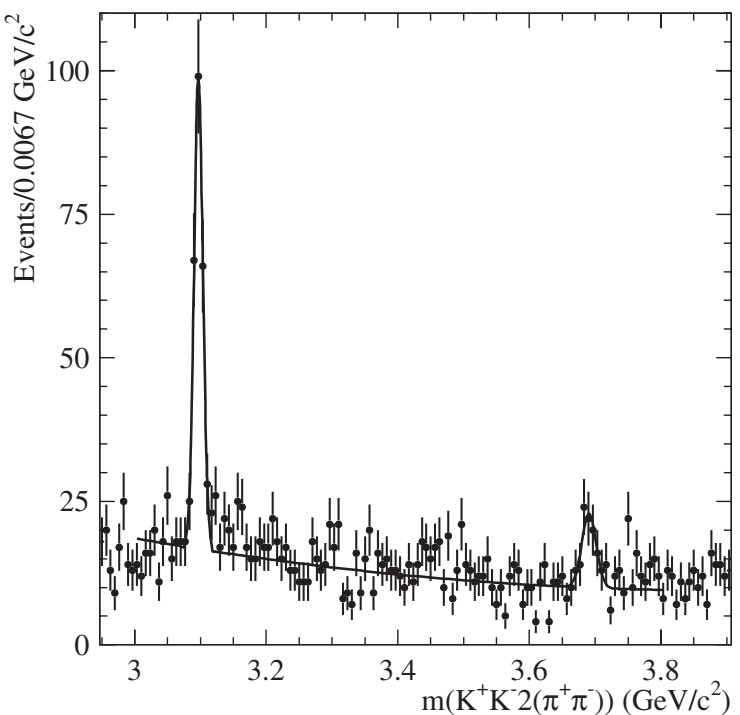

FIG. 29. The $K^{+} K^{-} 2\left(\pi^{+} \pi^{-}\right)$mass distribution for ISRproduced events in the mass region around the $J / \psi$ and $\psi(2 S)$; there are clear signals at the $J / \psi$ and $\psi(2 S)$ mass positions. The latter is dominated by $\psi(2 S) \rightarrow J / \psi \pi^{+} \pi^{-}$, with $J / \psi \rightarrow$ $K^{+} K^{-} \pi^{+} \pi^{-}$.

and using $B_{\psi(2 S) \rightarrow 6 \pi}=(1.5 \pm 1.0) \times 10^{-4}$ [24] only 5 events are expected-less than the expected statistical fluctuation in the observed number of $\psi(2 S)$ events. On the other hand, using $B_{\psi(2 S) \rightarrow J / \psi \pi^{+} \pi^{-}}=0.317 \pm 0.011$ [24] and the recently measured $B_{J / \psi \rightarrow 4 \pi}=(3.61 \pm$
$0.37) \times 10^{-3}$ [8], we estimate that $42 \pm 5$ events should be seen from the $\psi(2 S) \rightarrow J / \psi \pi^{+} \pi^{-} \rightarrow 3\left(\pi^{+} \pi^{-}\right)$decay chain. This estimate may be compared with a direct measurement of $46 \pm 8$ events, using data selected by taking events with four-pion invariant-mass combinations within $\pm 50 \mathrm{MeV} / \mathrm{c}^{2}$ of the $J / \psi$ mass and counting the number of $\psi(2 S)$ events in the six-pion mass distribution. Since the total number of events from the six-pion decay of the $\psi(2 S)$ is less than $1 \sigma$ larger than the number decaying through the $J / \psi \pi^{+} \pi^{-}$decay chain, no significant measurement can be made for the direct decay rate of $\psi(2 S) \rightarrow 3\left(\pi^{+} \pi^{-}\right)$from these data.

Figure 29 shows the $J / \psi$ and $\psi(2 S)$ signals in the $K^{+} K^{-} 2\left(\pi^{+} \pi^{-}\right)$mode. Background is not subtracted. The numbers of events under the Gaussian curves are $205 \pm 17$ and $51 \pm 11$, respectively. As shown in Sec. VI, $35 \pm 7 \mathrm{~J} / \psi$ events decay to the $\phi 4 \pi$ final state. The mass resolution is about $6.5 \mathrm{MeV} / \mathrm{c}^{2}$ for the $2 K 4 \pi$ channel. Using $B_{\psi(2 S) \rightarrow J / \psi \pi^{+} \pi^{-}}$and the recently measured $B_{J / \psi \rightarrow 2 K 2 \pi}=(6.2 \pm 0.7) \times 10^{-3}[8]$ we can expect $25 \pm$ 3 events due the decay chain $\psi(2 S) \rightarrow J / \psi \pi^{+} \pi^{-} \rightarrow$ $K^{+} K^{-} 2\left(\pi^{+} \pi^{-}\right)$. The difference of $26 \pm 13$ events between the total number of $\psi(2 S) \rightarrow K^{+} K^{-} 2\left(\pi^{+} \pi^{-}\right)$decays and this specific decay chain provides an estimate of direct $\psi(2 S) \rightarrow K^{+} K^{-} 2\left(\pi^{+} \pi^{-}\right)$decay.

Using the radiative correction factor 0.9 , ISR luminosity $d \mathcal{L} / d E=84.0 \mathrm{nb}^{-1} / \mathrm{MeV}$ at $\psi(2 S)$ mass, and $\epsilon_{2 K 4 \pi}$ from simulation with efficiency corrections, we obtain

$$
\begin{aligned}
B_{J / \psi \rightarrow K^{+} K^{-} 2\left(\pi^{+} \pi^{-}\right)} \cdot \sigma_{\text {int }}^{J / \psi} & =(66.2 \pm 5.5 \pm 4.0) \mathrm{nb} \mathrm{MeV}, \\
B_{J / \psi \rightarrow K^{+} K^{-} 2\left(\pi^{+} \pi^{-}\right)} \cdot \Gamma_{e e}^{J / \psi} & =(2.75 \pm 0.23 \pm 0.17) \times 10^{-2} \mathrm{keV}, \\
B_{J / \psi \rightarrow \phi(1020) 2\left(\pi^{+} \pi^{-}\right)} \cdot B_{\phi \rightarrow K^{+} K^{-}} \cdot \Gamma_{e e}^{J / \psi} & =(0.47 \pm 0.09 \pm 0.04) \times 10^{-2} \mathrm{keV}, \\
B_{\psi(2 S) \rightarrow K^{+} K^{-} 2\left(\pi^{+} \pi^{-}\right)} \cdot \Gamma_{e e}^{\psi(2 S)} & =(4.4 \pm 2.1 \pm 0.3) \times 10^{-3} \mathrm{keV} .
\end{aligned}
$$

The systematic errors are mainly due to the uncertainties in the acceptance and ISR luminosity.

Using the world average values for $\Gamma_{e e}^{J / \psi}, \Gamma_{e e}^{\psi(2 S)}$, and $B_{\phi \rightarrow K^{+} K^{-}}$[24], we calculate the branching fractions

$$
\begin{aligned}
B_{J / \psi \rightarrow 2 K 4 \pi} & =(5.09 \pm 0.42 \pm 0.35) \times 10^{-3}, \\
B_{J / \psi \rightarrow \phi 4 \pi} & =(1.77 \pm 0.35 \pm 0.12) \times 10^{-3}, \\
B_{\psi(2 S) \rightarrow 2 K 4 \pi} & =(2.1 \pm 1.0 \pm 0.2) \times 10^{-3},
\end{aligned}
$$

to be compared with the current world average values [24] of $(3.1 \pm 1.3) \times 10^{-3}$ for $J / \psi \rightarrow 2 K 4 \pi$ and $(1.60 \pm$ $0.32) \times 10^{-3}$ for $J / \psi \rightarrow \phi 4 \pi$. No earlier measurements are available for $\psi(2 S) \rightarrow K^{+} K^{-} 2\left(\pi^{+} \pi^{-}\right)$decays. The uncertainty in $\Gamma_{e e}^{J / \psi}$ has been added in quadrature to the systematic error estimate.
The observation of $J / \psi$ decaying into four-charged and two-neutral-pions gives a direct measurement of the $2\left(\pi^{+} \pi^{-} \pi^{0}\right)$-mass resolution and the absolute energy scale in this mode. The mass distribution after background subtraction is shown in Fig. 30. A fit with a Gaussian for the $J / \psi$ peak and a polynomial function for the continuum gives a mass resolution of $\sigma_{m\left(2\left(\pi^{+} \pi^{-} \pi^{0}\right)\right)}=12.7 \mathrm{MeV} / \mathrm{c}^{2}$. The central value of the mass lies $1.5 \mathrm{MeV} / \mathrm{c}^{2}$ above the PDG [24] value for the $J / \psi$ mass and gives an estimate of systematic uncertainty in absolute energy scale determined by the invariant mass of six pions. The observed mass resolution agrees with the simulation very well.

The $761 \pm 42$ events observed at the $J / \psi$ peak can be used to calculate the branching fraction for $J / \psi \rightarrow$ $2\left(\pi^{+} \pi^{-} \pi^{0}\right)$ as was done for $J / \psi \rightarrow 3\left(\pi^{+} \pi^{-}\right)$. We calculate the products: 


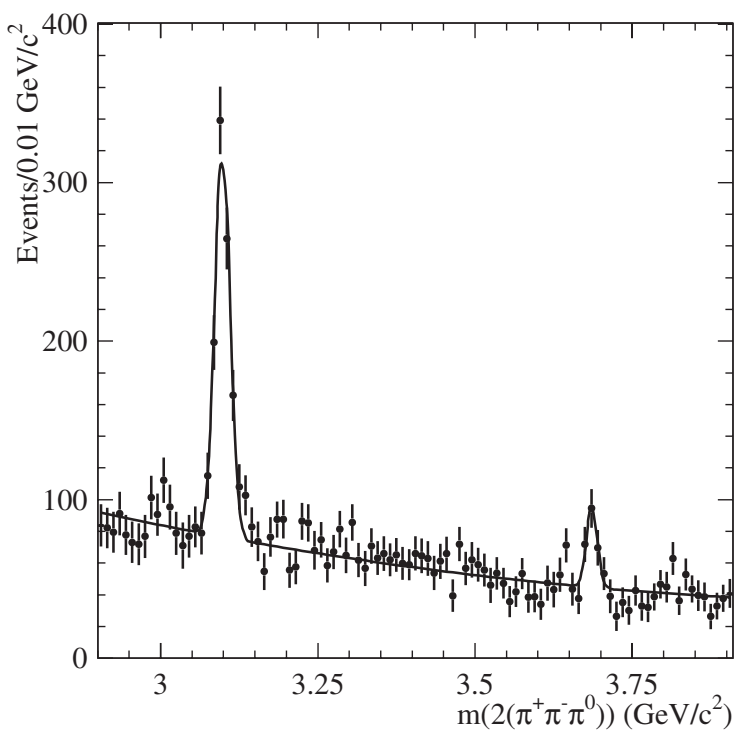

FIG. 30. The $2\left(\pi^{+} \pi^{-} \pi^{0}\right)$ mass distribution for ISR-produced events in the mass region around the $J / \psi$ and $\psi(2 S)$; there are clear signals at the $J / \psi$ and $\psi(2 S)$ mass positions.

$$
\begin{aligned}
B_{J / \psi \rightarrow 4 \pi 2 \pi^{0}} \cdot \sigma_{\text {int }}^{J / \psi} & =\frac{N\left(J / \psi \rightarrow 4 \pi 2 \pi^{0}\right)}{d \mathcal{L} / d E \cdot \epsilon_{\mathrm{MC}}} \\
& =215 \pm 12 \pm 24 \mathrm{nb} \mathrm{MeV} \\
B_{J / \psi \rightarrow 4 \pi 2 \pi^{0}} \cdot \Gamma_{e e}^{J / \psi} & =\frac{N\left(J / \psi \rightarrow 4 \pi 2 \pi^{0}\right) \cdot m_{J / \psi}^{2}}{6 \pi^{2} \cdot d \mathcal{L} / d E \cdot \epsilon_{\mathrm{MC}} \cdot C} \\
& =(8.9 \pm 0.5 \pm 1.0) \times 10^{-2} \mathrm{keV}
\end{aligned}
$$

where $\epsilon_{\mathrm{MC}}=0.060$ is the detection efficiency from simulation with the corrections and error discussed in Sec. V F. The subscript " $4 \pi 2 \pi^{0}$ " for branching fractions refers to the $2\left(\pi^{+} \pi^{-} \pi^{0}\right)$ final state exclusively.

Using $\Gamma_{e e}^{J / \psi}=5.40 \pm 0.18 \mathrm{keV}[24]$, we obtain the result $B_{J / \psi \rightarrow 4 \pi 2 \pi^{0}}=(1.65 \pm 0.10 \pm 0.18) \times 10^{-2}$. No entry for this branching fraction exists in PDG [24]. As was noted in Sec. VG, $170 \pm 24$ events are observed in the $J / \psi \rightarrow \omega \pi^{+} \pi^{-} \pi^{0}$ channel. This corresponds to the value

$$
B_{J / \psi \rightarrow \omega \pi^{+} \pi^{-} \pi^{0}} \cdot \Gamma_{e e}^{J / \psi}=(2.2 \pm 0.3 \pm 0.2) \times 10^{-2} \mathrm{keV},
$$

including the decay rate for $\omega \rightarrow \pi^{+} \pi^{-} \pi^{0}$. The corresponding fraction is $B_{J / \psi \rightarrow \omega \pi^{+} \pi^{-} \pi^{0}}=(0.41 \pm 0.06 \pm$ $0.04) \times 10^{-2}$, which is the first measurement of this branching fraction. There are also $13 \pm 3.6$ events from the $J / \psi \rightarrow \omega \eta$ decay. Taking into account the decay rates of $\omega$ and $\eta$ to $\pi^{+} \pi^{-} \pi^{0}$, we obtain $B_{J / \psi \rightarrow \omega \eta}=(1.47 \pm$ $0.41 \pm 0.15) \times 10^{-3}$, in agreement with the current PDG value $B_{J / \psi \rightarrow \omega \eta}=(1.58 \pm 0.16) \times 10^{-3}$.

The $\psi(2 S)$ peak can partly correspond to the decay chains $\psi(2 S) \rightarrow J / \psi \pi^{0} \pi^{0} \rightarrow 2\left(\pi^{+} \pi^{-} \pi^{0}\right)$ or $\psi(2 S) \rightarrow$ $J / \psi \pi^{+} \pi^{-} \rightarrow 2\left(\pi^{+} \pi^{-} \pi^{0}\right)$. Using $\quad B_{J / \psi \rightarrow 2\left(\pi^{+} \pi^{-}\right)}=$ $(3.61 \pm 0.37) \times 10^{-3}$ from Ref. [8], and assuming that

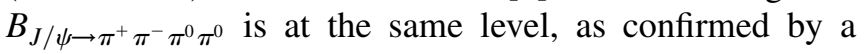

preliminary study of that channel, we estimate that $43 \pm$ 15 events from the above decay chains contribute to the $\psi(2 S)$ peak. The total number of $\psi(2 S)$ signal events extracted from a fit to a Gaussian distribution for the $\psi(2 S)$ peak and a polynomial function for the continuum is $128 \pm 20$. The $43 \pm 15$ events estimated above should be subtracted from this total to obtain an estimate for the number of direct $\psi(2 S) \rightarrow 2\left(\pi^{+} \pi^{-} \pi^{0}\right)$ decays.

Using $d \mathcal{L} / d E=84.0 \mathrm{nb}^{-1} / \mathrm{MeV}$ as the ISR luminosity at the $\psi(2 S)$ mass, $\epsilon_{\mathrm{MC}}=0.059$ as the detection efficiency from simulation with corrections and systematic error discussed in Sec. V F, and a 0.90 correction factor, we obtain

$$
\begin{aligned}
B_{\psi(2 S) \rightarrow 4 \pi 2 \pi^{0}} \cdot \sigma_{\mathrm{int}}^{\psi(2 S)} & =\frac{N\left(\psi(2 S) \rightarrow 4 \pi 2 \pi^{0}\right)}{d \mathcal{L} / d E \cdot \epsilon_{\mathrm{MC}}} \\
& =19.1 \pm 5.6 \pm 2.2 \mathrm{nb} \mathrm{MeV} \\
B_{\psi(2 S) \rightarrow 4 \pi 2 \pi^{0}} \cdot \Gamma_{e e}^{\psi(2 S)} & =\frac{N\left(\psi(2 S) \rightarrow 4 \pi 2 \pi^{0}\right) \cdot m_{\psi(2 S)}^{2}}{6 \pi^{2} \cdot d \mathcal{L} / d E \cdot \epsilon_{\mathrm{MC}} \cdot C} \\
& =(1.12 \pm 0.33 \pm 0.13) \times 10^{-2} \mathrm{keV} .
\end{aligned}
$$

Using the value of $\Gamma_{e e}^{\psi(2 S)}$ from the PDG [24] we calculate $B_{\psi(2 S) \rightarrow 4 \pi 2 \pi^{0}}=(5.3 \pm 1.6 \pm 0.6) \times 10^{-3}$. This is the first measurement of that branching ratio.

\section{THE $J / \psi$ PRODUCTION RATE AND CONTINUUM}

Having measured products of $\sigma_{\text {int }}^{J / \psi}$ and branching fractions for $J / \psi$ decaying to $6 \pi$ and $2 K 4 \pi$, it is interesting to compare them with the nonresonant cross sections (continuum) at that energy. Using a 2nd-order polynomial approximation of the cross sections from Tables I, III, and $\mathrm{V}$, around the $J / \psi$ peak within $\pm 0.4 \mathrm{GeV}$ (events from the peak are excluded), the following cross sections are obtained for the continuum at the $J / \psi$ mass:

$$
\begin{gathered}
\sigma_{6 \pi}=0.54 \pm 0.03 \mathrm{nb}, \quad \sigma_{4 \pi 2 \pi^{0}}=2.17 \pm 0.04 \mathrm{nb}, \\
\sigma_{2 K 4 \pi}=0.54 \pm 0.04 \mathrm{nb} .
\end{gathered}
$$

Table VI presents the ratios $B_{J / \psi \rightarrow f} \cdot \sigma_{\text {int }}^{J / \psi} / \sigma_{e^{+} e^{-} \rightarrow f}$ for $f=6 \pi, 2 K 4 \pi$. In these ratios most of the experimental systematic errors cancel. Also shown are the ratios for the

TABLE VI. Ratios of the $J / \psi$ partial production rates to continuum cross sections. The result for $\mu^{+} \mu^{-}$is from Ref. [6]. The results for $2\left(\pi^{+} \pi^{-}\right), K^{+} K^{-} \pi^{+} \pi^{-}$and $K^{+} K^{-} K^{+} K^{-}$are from Ref. [8].

\begin{tabular}{lc}
\hline \hline Final state, $f$ & $B_{J / \psi \rightarrow f} \cdot \sigma_{\text {int }}^{J / \psi} / \sigma_{e^{+} e^{-} \rightarrow f}(\mathrm{MeV})$ \\
\hline $3\left(\pi^{+} \pi^{-}\right)$ & $106 \pm 10$ \\
$2\left(\pi^{+} \pi^{-} \pi^{0}\right)$ & $99.1 \pm 6.5$ \\
$K^{+} K^{-} 2\left(\pi^{+} \pi^{-}\right)$ & $122 \pm 10$ \\
$2\left(\pi^{+} \pi^{-}\right)$ & $85.1 \pm 7.9$ \\
$K^{+} K^{-} \pi^{+} \pi^{-}$ & $166 \pm 19$ \\
$K^{+} K^{-} K^{+} K^{-}$ & $138 \pm 32$ \\
$\mu^{+} \mu^{-}$ & $84.12 \pm 0.67$ \\
\hline \hline
\end{tabular}


$J / \psi \rightarrow \mu^{+} \mu^{-}$final state [6], and $J / \psi \rightarrow 2\left(\pi^{+} \pi^{-}\right)$, $J / \psi \rightarrow K^{+} K^{-} \pi^{+} \pi^{-}$, and $J / \psi \rightarrow K^{+} K^{-} K^{+} K^{-}$[8].

The strong decay of the $J / \psi$ to an even number of pions is forbidden by $G$-parity conservation and therefore this decay is expected to be dominated by a single photon. No such suppression due to $G$ parity occurs for the strong decay of the $J / \psi$ for the other modes. The ratio obtained for both of the $6 \pi$ final states is close to that for $\mu^{+} \mu^{-}$and for $4 \pi$, indicating that the single-photon exchange dominates for the $J / \psi$ decays into these modes. For the $J / \psi$ decays to final states with kaons, the single-photon mechanism may be less dominant, as indicated by the significantly larger values of the ratios.

\section{SUMMARY}

The large $232 \mathrm{fb}^{-1}$ data sample accumulated near the $Y(4 S)$, together with the excellent resolution, chargedparticle identification, and open trigger of the BABAR detector, affords a unique opportunity to study the $3\left(\pi^{+} \pi^{-}\right), 2\left(\pi^{+} \pi^{-} \pi^{0}\right)$, and $K^{+} K^{-} 2\left(\pi^{+} \pi^{-}\right)$final states produced at low effective $e^{+} e^{-}$c.m. energy via ISR. Not only are the data samples extraordinarily large and well measured, but they do not suffer from the relative normalization uncertainties which seem to plague direct measurements from earlier storage rings such as DCI and SPEAR.

Since the luminosity and efficiencies are understood within 3\%-5\% accuracy, these data allow useful measurements of $R$, the ratio of the hadronic to di-muon cross sections, in the low $e^{+} e^{-}$energy regime, providing important input for calculating corrections needed for muon $g-2$ measurements. Cross section measurements for the reactions $e^{+} e^{-} \rightarrow 3\left(\pi^{+} \pi^{-}\right)$and $e^{+} e^{-} \rightarrow 2\left(\pi^{+} \pi^{-} \pi^{0}\right)$, with about $6 \%$ and $10 \%$ total systematic errors, respectively, are significant improvements over earlier measurements, while no earlier measurements exist for the reaction $e^{+} e^{-} \rightarrow K^{+} K^{-} 2\left(\pi^{+} \pi^{-}\right)$.

These final states also provide new information on hadron spectroscopy. Structure is observed around $19 \mathrm{GeV} / \mathrm{c}^{2}$, in both the $3\left(\pi^{+} \pi^{-}\right)$and $2\left(\pi^{+} \pi^{-} \pi^{0}\right)$ final states, which is similar to that previously observed by the DM2 and FOCUS experiments. A simple coherent model fits the structure well, and leads to the following parameters: $\quad m=1.87 \pm 0.02 \mathrm{GeV} / \mathrm{c}^{2}, \quad \Gamma=0.14 \pm 0.03 \mathrm{GeV}$ with a $-3 \pm 15^{\circ}$ relative phase to the continuum. However, these values are model dependent.

Resonance substructure is also seen in the intermediate mass combinations for these final states. The $\pi^{+} \pi^{-}$decay of the $\rho(770)$ dominates the substructure observed in the $3\left(\pi^{+} \pi^{-}\right)$final state, with little evidence for any other structures. A model with a single $\rho(770)$ describes the observed distributions very well. On the other hand, not only does the $2\left(\pi^{+} \pi^{-} \pi^{0}\right)$ final state display contributions from $\rho(770) 4 \pi$ but also from $\omega(782) \pi^{+} \pi^{-} \pi^{0}$ and $\omega(782) \eta$ final states. Structures that may correspond to $f_{0}(980), f_{0}(1370)$, and/or $f_{2}(1270)$ are also visible in intermediate mass combinations. A detailed understanding of this complex structure will probably require a full PWA using all of the six-pion final states.

Except for a structure around $1.6 \mathrm{GeV}$, the ratio of $e^{+} e^{-} \rightarrow 2\left(\pi^{+} \pi^{-} \pi^{0}\right)$ to $e^{+} e^{-} \rightarrow 3\left(\pi^{+} \pi^{-}\right)$cross sections is equal to $3.98 \pm 0.06 \pm 0.41$ over a wide range of c.m. energies. However, it is no longer flat and substantially smaller when the contribution from $\omega(782)$ decays to $\pi^{+} \pi^{-} \pi^{0}$ is excluded. These results can indicate and help us to understand more complex structures existing in the six-pion final states.

A resonancelike structure is seen in the $e^{+} e^{-} \rightarrow$ $\omega(782) \eta$ cross section around $1.6 \mathrm{GeV}$. A resonance fit gives $\quad m=1.645 \pm 0.008 \mathrm{GeV} / \mathrm{c}^{2}, \quad \Gamma=0.114 \pm$ $0.014 \mathrm{GeV}$ and a peak cross section $\sigma_{0}=3.08 \pm$ $0.33 \mathrm{nb}$. This object might be associated with the $\omega(1650)$, for which a $\omega(782) \eta$ final state has been seen [24], but the width measured here is substantially smaller. The observed parameter values are actually much closer to those of the $\phi(1680)$ [24], but there are no observations for an $\omega(782) \eta$ decay mode of the $\phi(1680)$.

The energy dependence of the cross section for the reaction $e^{+} e^{-} \rightarrow K^{+} K^{-} 2\left(\pi^{+} \pi^{-}\right)$from threshold to $4.5 \mathrm{GeV}$ is measured with about $15 \%$ systematic uncertainty. There is clear evidence for resonance substructure including the $K^{* 0}(892)$ and $\phi$.

These data also allow a study of $J / \psi$ and $\psi(2 S)$ production, and the measurement of the product of decay branching fractions and the $e^{+} e^{-}$width of the $J / \psi$ with the best accuracy to date. The results are as follows:

$$
\begin{aligned}
& B_{J / \psi \rightarrow 3\left(\pi^{+} \pi^{-}\right)} \cdot \Gamma_{e e}^{J / \psi}=(2.37 \pm 0.16 \pm 0.14) \times 10^{-2} \mathrm{keV}, \\
& B_{J / \psi \rightarrow K^{+} K^{-} 2\left(\pi^{+} \pi^{-}\right)} \cdot \Gamma_{e e}^{J / \psi}=(2.75 \pm 0.23 \pm 0.17) \times 10^{-2} \mathrm{keV}, \\
& B_{J / \psi \rightarrow \phi(1020) 2\left(\pi^{+} \pi^{-}\right)} \cdot B_{\phi \rightarrow K^{+} K^{-}} \cdot \Gamma_{e e}^{J / \psi}=(0.47 \pm 0.09 \pm 0.03) \times 10^{-2} \mathrm{keV} \text {, } \\
& B_{J / \psi \rightarrow 2\left(\pi^{+} \pi^{-} \pi^{0}\right)} \cdot \Gamma_{e e}^{J / \psi}=(8.9 \pm 0.5 \pm 1.0) \times 10^{-2} \mathrm{keV}, \\
& B_{J / \psi \rightarrow \omega \pi^{+} \pi^{-} \pi^{0}} \cdot \Gamma_{e e}^{J / \psi}=(2.2 \pm 0.3 \pm 0.2) \times 10^{-2} \mathrm{keV}, \\
& B_{\psi(2 S) \rightarrow 2\left(\pi^{+} \pi^{-} \pi^{0}\right)} \cdot \Gamma_{e e}^{\psi(2 S)}=(1.12 \pm 0.33 \pm 0.12) \times 10^{-2} \mathrm{keV}, \\
& B_{\psi(2 S) \rightarrow K^{+} K^{-} 2\left(\pi^{+} \pi^{-}\right)} \cdot \Gamma_{e e}^{\psi(2 S)}=(4.4 \pm 2.1 \pm 0.3) \times 10^{-3} \mathrm{keV} \text {. }
\end{aligned}
$$


Dominance of the single-photon decay mechanism for $J / \psi \rightarrow 3\left(\pi^{+} \pi^{-}\right)$and $J / \psi \rightarrow 2\left(\pi^{+} \pi^{-} \pi^{0}\right)$ decays has been demonstrated by comparison with the continuum cross sections for $e^{+} e^{-} \rightarrow 3\left(\pi^{+} \pi^{-}\right)$and $e^{+} e^{-} \rightarrow$ $2\left(\pi^{+} \pi^{-} \pi^{0}\right)$.

\section{ACKNOWLEDGMENTS}

We are grateful for the extraordinary contributions of our PEP-II colleagues in achieving the excellent luminosity and machine conditions that have made this work possible. The success of this project also relies critically on the expertise and dedication of the computing organizations that support $B A B A R$. The collaborating institutions wish to thank SLAC for its support and the kind hospitality extended to them. This work is supported by the US Department of Energy and National Science Foundation, the Natural Sciences and Engineering Research Council
(Canada), Institute of High Energy Physics (China), the Commissariat à l'Energie Atomique and Institut National de Physique Nucléaire et de Physique des Particules (France), the Bundesministerium für Bildung und Forschung and Deutsche Forschungsgemeinschaft (Germany), the Istituto Nazionale di Fisica Nucleare (Italy), the Foundation for Fundamental Research on Matter (The Netherlands), the Research Council of Norway, the Ministry of Science and Technology of the Russian Federation, and the Particle Physics and Astronomy Research Council (United Kingdom). Individuals have received support from CONACyT (Mexico), the Marie-Curie Intra European Fellowship program (European Union), the A. P. Sloan Foundation, the Research Corporation, and the Alexander von Humboldt Foundation.
[1] V. N. Baier and V. S. Fadin, Phys. Lett. B 27, 223 (1968).

[2] A. B. Arbuzov et al., J. High Energy Phys. 12 (1998) 009.

[3] S. Binner, J. H. Kühn, and K. Melnikov, Phys. Lett. B 459, 279 (1999).

[4] M. Benayoun et al., Mod. Phys. Lett. A 14, 2605 (1999).

[5] M. Davier, S. Eidelman, A. Hocker, and Z. Zhang, Eur. Phys. J. C 31, 503 (2003).

[6] B. Aubert et al. (BABAR Collaboration), Phys. Rev. D 69, 011103 (2004).

[7] B. Aubert et al. (BABAR Collaboration), Phys. Rev. D 70, 072004 (2004).

[8] B. Aubert et al. (BABAR Collaboration), Phys. Rev. D 71, 052001 (2005).

[9] B. Aubert et al. (BABAR Collaboration), Nucl. Instrum. Methods Phys. Res., Sect. A 479, 1 (2002).

[10] H. Czyż and J. H. Kühn, Eur. Phys. J. C 18, 497 (2001).

[11] A. B. Arbuzov et al., J. High Energy Phys. 10 (1997) 001.

[12] M. Caffo, H. Czyż, and E. Remiddi, Nuovo Cimento Soc. Ital. Fis. A 110, 515 (1997); Phys. Lett. B 327, 369 (1994).

[13] E. Barberio, B. van Eijk, and Z. Was, Comput. Phys. Commun. 66, 115 (1991).

[14] S. Agostinelli et al. (GEANT4 Collaboration), Nucl. Instrum. Methods Phys. Res., Sect. A 506, 250 (2003).

[15] T. Sjostrand, Comput. Phys. Commun. 82, 74 (1994).
[16] S. Jadach and Z. Was, Comput. Phys. Commun. 85, 453 (1995).

[17] S. Eidelman and F. Jegerlehner, Z. Phys. C 67, 585 (1995).

[18] R. Baldini et al., in Proceedings of the "Fenice" Workshop, Frascati, 1988; A.B. Clegg and A. Donnachie, Z. Phys. C 45, 677 (1990); M. R. Whalley, J. Phys. G 29, A1 (2003).

[19] B. Jean-Marie et al., SLAC Report No. SLAC-PUB-1711, 1976 (to be published).

[20] P. L. Frabetti et al. [E687 (FOCUS) Collaboration], Phys. Lett. B 514, 240 (2001).

[21] M. Grilli et al., Nuovo Cimento Soc. Ital. Fis. A 13, 593 (1973).

[22] C. Bacci et al., Nucl. Phys. B184, 31 (1981).

[23] B. Esposito et al., Lett. Nuovo Cimento Soc. Ital. Fis. 31, 445 (1981).

[24] Review of Particle Physics, S. Eidelman et al., Phys. Lett. B 592, 1 (2004).

[25] N. N. Achasov and A. A. Kozhevnikov, Phys. Rev. D 55, 2663 (1997).

[26] M. Jacob and R. Slansky, Phys. Lett. B 37, 408 (1971); Phys. Rev. D 5, 1847 (1972).

[27] P. L. Frabetti et al., Phys. Lett. B 578, 290 (2004). 